\title{
Structural and functional studies of the first tripartite protein complex at the Trypanosoma brucei flagellar pocket collar
}

Charlotte Isch ${ }^{1, a}$, Paul Majneri2,a, Nicolas Landrein ${ }^{1, a}$, Yulia Pivovarova², Johannes Lesigang², Florian Lauruol ${ }^{1}$, Derrick. R. Robinson ${ }^{1}$, Gang Dong ${ }^{2, b}$, Mélanie Bonhivers ${ }^{1, b}$

${ }^{1}$ Univ. Bordeaux, CNRS, Microbiologie Fondamentale et Pathogénicité, UMR 5234, F-33000 Bordeaux, France

${ }^{2}$ Max Perutz Labs, Vienna BioCenter, Medical University of Vienna, Vienna, Austria ${ }^{a}$ co-first authors

${ }^{\mathrm{b}}$ co-corresponding authors

*For correspondance:

Mélanie Bonhivers, CNRS UMR5234, Université de Bordeaux, 146 rue Léo Saignat, 33076, Bordeaux, France.melanie.bonhivers@u-bordeaux.fr

Gang Dong, Max Perutz Labs, Vienna BioCenter, Medical University of Vienna, Dr. BohrGasse 9, 1030 Vienna, Austria.gang.dong@meduniwien.ac.at

Short title: A tripartite interaction at the T. brucei Flagellar Pocket Collar 


\section{Abstract}

The flagellar pocket (FP) is the only endo- and exocytic organelle in most trypanosomes and, as such, is essential throughout the life cycle of the parasite. The neck of the FP is maintained enclosed around the flagellum via the flagellar pocket collar (FPC). The FPC is a macromolecular cytoskeletal structure and is essential for the formation of the FP and cytokinesis. FPC biogenesis and structure are poorly understood, mainly due to the lack of information on FPC composition. To date, only two FPC proteins, BILBO1 and FPC4, have been characterized. BILBO1 forms a molecular skeleton upon which other FPC proteins can, theoretically, dock onto. We previously identified FPC4 as the first BILBO1 interacting partner and demonstrated that its C-terminal domain interacts with the BILBO1 N-terminal domain (NTD). Here, we report the characterization of a new FPC component and BILBO1 partner protein, BILBO2 (Tb927.6.3240) by yeast two-hybrid screen, bioinformatics, functional and structural studies. We show that BILBO2 colocalizes with BILBO1 and can modulate the shape of the BILBO1 filament by interacting with the BILBO1 EF-hand domains. Further, we demonstrate that BILBO1 and BILBO2 share a homologous NTD domain and that both domains interact with FPC4. We have determined a $1.9 \AA$ A resolution crystal structure of the BILBO2 NTD in complex with the FPC4 BILBO1-binding domain. Together with mutational analyses, our studies reveal key residues for the function of the BILBO2 NTD and its interaction with FPC4 and evidenced a tripartite interaction between BILBO1, BILBO2, and FPC4. Our work sheds light on the first atomic structure of an FPC protein complex and represents a significant step in deciphering the FPC function in Trypanosoma brucei and other pathogenic kinetoplastids. 


\section{Author summary}

Trypanosomes belong to a group of zoonotic, protist, parasites that are found in Africa, Asia, South America, and Europe and are responsible for severe human and animal diseases. They all have a common structure called the flagellar pocket (FP). In most trypanosomes, all macromolecular exchanges between the trypanosome and the environment occur via the FP. The FP is thus essential for cell viability and evading the host immune response. We have been studying the flagellar pocket collar (FPC), an enigmatic macromolecular structure at the neck of the FP, and demonstrated its essentiality for the biogenesis of the FP. We demonstrated that BILBO1 is an essential protein of the FPC that interacts with other proteins including a microtubule-binding protein FPC4.

Here we identify another bona fide FPC protein, BILBO2, so named because of close similarity with BILBO1 in protein organization and functional domains. We demonstrate that BILBO1 and BILBO2 share a common N-terminal domain involved in the interaction with FPC4, and illustrate a tripartite interaction between BILBO1, BILBO2, and FPC4. Our study also provides the first atomic view of two FPC components. These data represent an additional step in deciphering the FPC structure and function in T. brucei. 


\section{Introduction}

Trypanosomatids include many parasites of major medical and economic importance that cause several of the 20 World Health Organization's listed neglected tropical diseases. These flagellated parasites share several unique features: a single mitochondrion with its compact genome (the kinetoplast, K), a flagellar pocket (FP), and a microtubule-based cytoskeleton to maintain cell shape and flagellar motility that plays crucial roles in life and cell cycle [1]. The FP is an invagination of the plasma membrane enclosing the base of the flagellum. In most trypanosomes, endo- and exocytosis occur exclusively through the FP. It thus provides the sole surface for numerous important receptors making them inaccessible for components of the innate immune system of the host. Moreover, the FP is responsible for sorting all parasite surface glycoproteins targeted to, or recycling from, the pellicular membrane and for removal of host antibodies from the cell surface. As such, the FP is a key player in protein trafficking, cell signalling and immune evasion [2]. Because it is hidden from the cell surface and sequesters important receptors, the FP is an attractive drug target. However, it has not been exploited as such because structural components of this organelle are still poorly characterized.

During its life cycle, $T$. brucei is transmitted to the mammalian host via a blood meal of an infected tsetse fly. The parasite differentiates to several different forms in the insect and the mammalian host, among them the procyclic form (PCF) in the fly's midgut, and the bloodstream form (BSF) in the mammalian bloodstream. Organelle positioning and segregation during the cell and parasite cycle show a high degree of coordination and control [3].

The shape of the trypanosome cell is maintained by a microtubule-based corset and by a flagellum laterally attached along the cell body. The flagellum is involved in cell mobility, kinetoplast segregation, and signal transduction [1]. It extends from the mature basal body (BB, tethered to the kinetoplast), and exits the cell through the FP. It then runs along the length of the cell while remaining attached to the cell body via the flagellum attachment zone (FAZ). Four specialized microtubules (the microtubule quartet, MTQ) nucleate at the BBs and extend around the FP, insert into the microtubule corset, and run as part of the cytoplasmic portion of the FAZ as far as to the anterior end of the cell body. The bulb-like FP is maintained by a 
ring-like cytoskeletal structure, the flagellar pocket collar (FPC), which encircles the neck of the FP around the exit site of flagellum beneath the cell surface $[4,5]$. The FPC is a complex structure, and in addition to its attachment to the flagellum, it is also attached to the microtubule cytoskeleton. Overlapping with the FPC is the hook complex (HC), a cytoskeletonassociated structure that is superimposed on top of the FPC throughout the cell cycle. The MTQ threads between these two structures [6-8].

BILBO1 is the first identified FPC protein, with an indispensable role for the parasite [4]. RNA interference (RNAi) knockdown of BILBO1 in PCF cells prevents the biogenesis of a new FPC, a new FP, and a new FAZ, suggesting that the FPC is required for the biogenesis of numerous structures and their functions in the cell. In BILBO1 RNAi cells, the newly formed flagellum locates at the extended posterior end of the cell and is detached from the cell body. Furthermore, knockdown of BILBO1 is lethal in both PCF and BSF cells.

BILBO1 is a modular protein with four structural domains $[9,10]$ (Fig $1 \mathrm{~A})$. The globular $\mathrm{N}$ terminal domain (NTD) is followed by two calcium-binding EF-hand motifs (EFhs), a central coiled-coil domain (CCD), and a C-terminal leucine zipper (LZ). The LZ is necessary but not sufficient for FPC targeting of BILBO1. The CCD allows the formation of filaments by the formation of antiparallel dimers that can extend into a polymer by the interdimer interaction between adjacent LZs. Indeed, BILBO1 was shown to form micrometre-long polymers and helical structures in vivo, in vitro and in a mammalian cell environment [4,9-12].

The reported high-resolution NMR and crystal structures of the BILBO1-NTD demonstrate that it unexpectedly adopts a ubiquitin-like fold $[13,14]$. The C-terminal tail of the NTD is wellfolded and rigidly wraps around the distal end of the elongated core structure. This tail helps to form a well-defined horseshoe-like pocket that harbours multiple highly conserved aromatic residues. On one side of the hydrophobic pocket, a gap is formed that leads to a pronounced negative trench at the bottom of the structure. Mutation of key residues within the pocket affect cell viability and impair the BILBO1 function in trypanosomes. Further, abolishing the $\mathrm{Ca}^{2+}$-binding ability of the EFh influences the shape and length of the polymers of BILBO1, disrupts the FPC structure, and affects trypanosome cell viability $[9,10,15]$. Deletion of both globular domains (i.e. NTD and EFh) leads to shorter polymers than those formed by the full-length BILBO1 [9], suggesting their role in facilitating inter-dimeric interactions.

To date, a handful of FPC or FPC-associated proteins have been identified (BILBO1, FPC5, 
FPC4, Tb927.11.5640) $[4,10,15,16]$ but only two, BILBO1 and FPC4, have been characterized at the molecular level $[4,9,13,15]$. We have previously demonstrated that FPC4 binds to microtubules via its N-terminus, and interacts with the NTD of BILBO1 via its C-terminal domain (CTD). In T. brucei, FPC4 localizes at the interface between the FPC and the HC, suggesting a role in linking the FPC-HC-MTQ structures. Static light scattering experiments demonstrated that the BILBO1-NTD and the FPC4-CTD form a stable binary complex [14]. Using a combination of biophysical and cell biology approaches, we have shown that FPC4 binds to the horseshoe-like aromatic patch of the BILBO1-NTD [15].

Despite extensive structural and functional studies on BILBO1, the mechanisms underlying the macro-molecular assembly and biogenesis of the FPC remain elusive, mainly due to the poor knowledge of its molecular composition and assembly. Here we report the identification and characterization of Tb927.6.3240, a novel BILBO1-partner protein at the FPC. We show that it is a bona fide FPC protein with an N-terminal domain structurally and functionally homologous to that of BILBO1. We thus named it BILBO2. We demonstrate here that similarly to BILBO1, BILBO2 interacts with the CTD of FPC4 via its NTD. We have further determined a 1.9-Å resolution crystal structure of the BILBO2-NTD/FPC4-CTD complex, which provides a clear view of how the extended FPC4 polypeptide docks into the horseshoe-like aromatic pocket on BILBO2. This is the first molecular structure of the FPC protein complex and represents a significant step toward deciphering the FPC interactome in T. brucei. Overall, our data identify a common module in two different FPC components that are essential in FPC biogenesis and cell viability. 


\section{Results}

\section{BILBO2 (Tb927.6.3240) is a bona fide FPC protein interacting with BILBO1}

Using BILBO1 as a bait in a T. brucei 927 genomic yeast two-hybrid screen (Y2H), we identified FPC4 as a BILBO1 binding partner [15]. We also identified a kinetoplastid-specific protein of 271 amino acids, Tb927.6.3240 [17], which we named BILBO2 based on its shared characteristics with BILBO1 (Fig 1A).

The interaction between $\mathrm{BILBO} 1$ and $\mathrm{BILBO} 2$ identified during the $\mathrm{Y} 2 \mathrm{H}$ genomic screen was further narrowed down to sub-domains of the two proteins by serial truncation analyses. $\mathrm{Y} 2 \mathrm{H}$ assays demonstrated that aa 151-271 domain of BILBO2 is both necessary and sufficient for the BILBO1-BILBO2 interaction, and was therefore named BILBO1-binding domain (B1BD) (Fig 1B). Interestingly, the $\mathrm{Y} 2 \mathrm{H}$ assay also suggests that $\mathrm{BILBO} 2$ interacts with other $\mathrm{BILBO} 2$ molecules (Fig 1B, BILBO2 x BILBO2).

We previously used the U-2 OS mammalian heterologous expression system as a tool to characterize the BILBO1 polymers and its binding to FPC4 $[10,15]$. Similarly, we further characterize the BILBO1 domain involved in the interaction with BILBO2. Individual expression of BILBO1 and of HA-tagged BILBO2 ( $\mathrm{HA} B$ LBO2) followed by whole-cell immunodetection shows the BILBO1 polymers (Fig 2Aa) and a cytosolic localization for ${ }_{\mathrm{HA}} \mathrm{BILBO2}$ (Fig 2Ab). However, co-expression of BILBO1 and ${ }_{\mathrm{HA}} \mathrm{BILBO} 2$ confirmed the interaction between the two proteins as shown by the localization of BILBO2 onto the BILBO1 polymers (Fig 2AC).

To further characterize the BILBO1-BILBO2 interaction, we individually expressed in U-2 OS

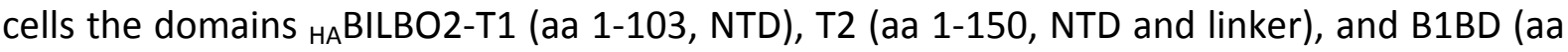
151-271) (S1 Fig) individually or in combination with BILBO1 (Fig 2A d-f). Immunolabelling of BILBO1 and ${ }_{H A} B I L B O 2$ domains confirms that BILBO2 binds to BILBO1 specifically via its BILBO1-BD (B1BD) and not via its T1 or T2 domains. BILBO1-BILBO2 interaction is resistant to non-ionic detergent extraction as observed in the extracted cytoskeletons upon co-expression of $\mathrm{BILBO}_{\mathrm{GFP}}$ and ${ }_{\mathrm{HA}} \mathrm{BILBO2}$ (Fig 2Ba). We further used the polymerizing properties of the BILBO1 truncations $\mathrm{T3}_{\text {GFP }}$ and T4 $4_{\text {GFP }}$ (Fig. 1A) [10] to identify the BILBO1 domain involved in the interaction using detergent-extracted cells (Fig 2B). Thereby, we show that BILBO2 interacts with BILBO1-T3 (Fig 2B, b) but not with T4 (Fig 2B, c) suggesting that BILBO2 interacts with the BILBO1 EF-hand domain and that BILBO2-B1BD is sufficient to bind to BILBO1-T3 (Fig 2B, d). 
Mutation of key residues for calcium-binding of BILBO1-EFh1 (mEFh1) or EFh2 (mEFh2) induces conformational changes in the shape of the BILBO1 polymers in U-2 OS cells and trypanosomes, suggesting that the holo or apo calcium status of the EF-hands may regulate BILBO1 assembly $[9,10]$. We thus wondered if the BILBO1-BILBO2 interaction is modulated by calcium. As previously reported [10], expression of mutated EFh1 (mEFh1) induced the formation of highly compact BILBO1 polymer structures, while mEFh2 and mEFh1+2 induced helical/ball-like structures (Fig 2C, a-c). We further show here that deletion of both EF-hands $(\Delta \mathrm{EFh} 1+2)$ also induce the formation of short polymers (Fig $2 \mathrm{C}, \mathrm{d})$. None of the EF-hand mutations affected BILBO2 binding (Fig $2 \mathrm{C}$, e-g). However, their deletion $(\Delta \mathrm{EFh} 1+2)$ abolished the BILBO1-BILBO2 interaction (Fig 2C, h). Interestingly, we observed that, when bound to BILBO2 (Fig 2C, e), BILBO1-mEFh1 formed short linear filaments contrary to the very compact or aggregated structures in the absence of BILBO2 (a). Similarly, mEFh2, when bound to BILBO2 (Fig 2C, f), also formed linear filaments that were not observed when BILBO1-mEFh2 is expressed alone (Fig $2 \mathrm{C}, \mathrm{b}$ ).

In a global quantitative phosphoproteomic analysis, serine 171 and 173 of BILBO2 were shown phosphorylated in vivo [18]. Because these residues belong to the BILBO1-BD of BILBO2 (Fig 1A), we postulated that their mutation might affect binding. We co-expressed in U-2 OS cells the non-phosphorylable (S171A, S173A, S171A+S173A) or phosphomimetic (S171D, S173D, S171D+S173D) mutant forms of ${ }_{H A} B I L B O 2$ with BILBO1. None of these mutations impaired the co-localization of BILBO1 and BILBO2, suggesting that they are not directly involved in the interaction. Nevertheless, the binding affinity might be subtly changed in vivo.

Taken together, these data identify BILBO2 as a novel BILBO1-binding protein that plays a role in the oligomerization of BILBO1 and may regulate BILBO1-mediated FPC assembly or function in the parasite.

\section{BILBO2 localizes at the FPC primarily via its BILBO1-binding domain}

To determine the localization of BILBO2 in T. brucei, we generated a guinea pig BILBO2specific antibody and cell lines expressing epitope-tagged fusion BILBO2 (epitopeBILBO2) using the PPOTV7 vector series for endogenous tagging [19] and controlled that the tag has no effect on cell growth (S2 Fig. A). Co-labelling of BILBO1 and BILBO2 on T. brucei detergent-extracted cells (cytoskeleton, $\mathrm{CSK}$ ) revealed that $\mathrm{BILBO} 2$ and ${ }_{\mathrm{TY} 1} \mathrm{BILBO} 2$ co-localized with $\mathrm{BILBO} 1$ at every 
stage of the cell cycle of PCF (Fig 3A, B) and of BSF (S2 Fig. B). To identify the FPC targeting domain of BILBO2, we generated PCF cell lines inducible for the ectopic expression BILBO2 ${ }_{\mathrm{HA}}$ and truncations BILBO2-T1 $1_{\mathrm{HA}}, \mathrm{BILBO2}-\mathrm{T} 2_{\mathrm{HA}}$ and BILBO2-B1BD $\mathrm{HA}$. No dominant-negative phenotype or change in cell growth was observed after expression of any of these constructs (S3 Fig). Protein localization was assessed by immunofluorescence on CSK, which showed that both $\mathrm{BILBO}_{\mathrm{HA}}$ and BILBO2-B1BD $\mathrm{HA}$ were detected at the FPC (Fig $3 \mathrm{C}$ ). The BILBO2-T1 $1_{\mathrm{HA}}$ domain was removed during extraction, suggesting that T1 alone is not sufficient for FPC binding. Similarly, BILBO2-T2 ${ }_{\mathrm{HA}}$ was mostly extracted, however, very weak but consistent labelling was observed at the FPC (asterisk), suggesting that the linker domain between the BILBO2-NTD and its B1BD is involved in FPC binding via another partner or that the $\mathrm{T} 1$ construct was too short for proper function (Fig. 3C).

Using DAPI as a marker for cell cycle stages (number of kinetoplasts and nuclei) and anti-

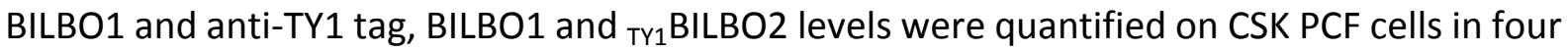
cell cycle stages using Image (Fig 3D). The fluorescence intensity of BILBO1 remained constant at each FPC (old and new) during the cell cycle. Interestingly, the intensity of BILBO2 labelling varied dramatically during the cell cycle, with approximately $50 \%$ reduction in cells with 1 kinetoplast, 1 nucleus, but 2 FPCs (1K1N-2FPC, 2K1N-2FPC) in both the new and the old FPC. When the cell reaches cytokinesis, the BILBO2 levels were almost equivalent to those at the beginning of the cell cycle. This suggests that BILBO2 expression is cell-cycle regulated in a different way to BILBO1.

To further assess BILBO2 function in PCF and BSF of trypanosomes, we generated BILBO2 RNAi knockdown cell lines in the endogenously tagged background using the tetracyclineinducible stem-loop RNAi system [20]. PCF and BSF cell growth was not affected after several days of induction, and no morphological phenotypes were observed even though specific reduction of $\mathrm{BILBO} 2$ expression was observed by western blotting (S4 Fig). However, several attempts to generate BILBO2 knock-out PCF and BSF cell lines failed. It is noteworthy that previous RNAi screen indicated that depletion of BILBO2 causes a deleterious effect on cell viability in both trypanosome life forms [21]. These data suggest that BILBO2 might play a critical role in the cell, whereas trace amount of proteins left in RNAi knockdown may have 
been sufficient to carry out such function and thus showed no defects in cell growthor other phenotypes.

\section{Localization of BILBO2 to the FPC depends on BILBO1}

We have reported previously that, upon RNAi knockdown of BILBO1, PCF cells display a cell cycle arrest in $2 \mathrm{~K} 2 \mathrm{~N}$ stage and a detached new flagellum. Furthermore, the biogenesis of the new FAZ, FP, and FPC are also prevented, which eventually lead to cell death [4]. In addition, some of the FPC4 and MORN1 (a HC protein) proteins are mis-localized within the new detached flagellum [15]. Therefore to analyse the fate of BILBO2 in the absence of a new FP and a new FPC, we generated an inducible BILBO1 RNAi cell line expressing ${ }_{\mathrm{TY} 1} \mathrm{BILBO2}$ (Fig 4). BILBO1 RNAi-induced cells stopped proliferating after $48 \mathrm{~h}$, and displayed a $2 \mathrm{~K} 2 \mathrm{~N}$ growth arrest and detached new flagella (Fig 4A, B). Immunofluorescence on whole cells revealed that after $48 \mathrm{~h}$ of induction BILBO2 was neither detected at the old flagellum nor at the new flagellum. Instead, a punctate cytosolic pool was observed (Fig 4B). Interestingly, westernblotting quantification showed that the CSK-associated pool of BILBO2 decreased by $5.3 \times$ fold over the time-course of BILBO1 RNAi knockdown, while the total pool of BILBO2 (WC) increased by $2.6 \times$ fold (Fig 4C, D). These data show that during BILBO1 knockdown, and thus in the absence of a new FPC, BILBO2 is still neo-synthetized but is not associated with the cytoskeleton anymore.

\section{The N-terminal domain of BILBO2 is homologous to the BILBO1 N-terminal domain}

BILBO1 is a multi-domain protein and its NTD interacts with FPC4-CTD via key residues in a conserved surface patch that are involved in the BILBO1 function [15]. Database mining for proteins sharing a domain homologous to BILBO1-NTD identified the $\mathrm{N}$-terminal domain of BILBO2. Alignment of BILBO1 and BILBO2 sequences revealed an overall similarity of $19 \%$ between the full-length proteins. However, the identity and similarity reach $32 \%$ and $38 \%$ respectively between their NTD (Fig 5A). Importantly, the two residues (Y64, W71), which were previously shown to play a critical role in BILBO1's interaction with FPC4 and cell viability $[10,13,15]$, are conserved or identical in BILBO2 (i.e. F63, W70). However, apart from its NTD and B1BD, no other structural or functional domains were identified in BILBO2. 
We hypothesized that if the NTD of BILBO1 and BILBO2 are functionally similar, they could be exchanged without affecting the function of either protein. Chimeric BILBO1 and BILBO2 proteins with exchanged NTDs, namely ${ }_{\text {ch BILBO1-BILBO2 }}{ }_{\text {HA }}$ (BILBO1 aa1-118 fused to BILBO2 aa111-271) and ${ }_{C h} B I L B O 2-B I L B O 1_{H A}$ (BILBO2 aa1-110 fused to BILBO1 aa119-587), were ectopically expressed in PCF T. brucei (Fig 5B). It is important to note that, as previously described [10], ectopic expression of WT BILBO1, T3 or T4 is lethal due to excessive polymer formation induced by the CCD and LZ domains [10]. Thus, expression of any construct containing BILBO1 CCD-LZ results in a growth phenotype and lethality and explains the growth phenotype that occurs when $\mathrm{Ch}_{\mathrm{BILBO}}-\mathrm{BILBO}_{\mathrm{HA}}$ is expressed (S5 Fig). Nevertheless, using an anti-BILBO1 antibody recognizing aa1-110 of both BILBO1 and the chimeric ${ }_{\mathrm{Ch}} \mathrm{BILBO1-BILBO} 2_{\mathrm{HA}}$ proteins, and an anti-HA antibody recognizing the chimeric proteins only, we immunolocalized endogenous BILBO1 and both chimeric proteins in detergent-extracted cells. Both

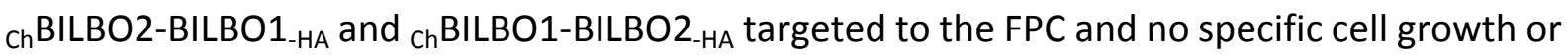
morphology phenotype was observed (Fig 5B, S5). This supports the hypothesis that the NTDs share a similar function as they can compensate for each other in both chimera. This is imperative because we have demonstrated that minor changes in BILBO1-NTD, such as the W71A mutant, were lethal [14].

\section{BILBO2 is an FPC protein as well as a FPC4 interacting partner and binds to FPC4 via its N- terminal domain}

We previously demonstrated that FPC4 binds to microtubules via its N-terminus, and interacts with BILBO1 via its C-terminal region (FPC4-B1BD), suggesting a role in linking the FPC-HC-MTQ structure. Using a combination of site-directed mutagenesis, biophysical and cell biology approaches, we also showed that FPC4 binds to a conserved hydrophobic patch on the BILBO1-NTD surface patch. Further, static light scattering experiments demonstrated that the BILBO1-NTD and the FPC4-B1BD form a stable binary complex [15]. The recent highresolution crystal structure of BILBO1-NTD and mutagenesis studies revealed that FPC4 interacts with BILBO1 by mainly contacting three aromatic residues W71, Y87, and F89 in the centre of the conserved hydrophobic patch [14].

Based on our data showing that the NTDs of BILBO1 and BILBO2 function similarly and the high homology shared between them, we checked whether BILBO2-NTD also forms a stable 
complex with FPC4-B1BD. We first tested the interaction between BILBO2 and FPC4 (fulllength or truncations) by $\mathrm{Y} 2 \mathrm{H}$ (Fig $6 \mathrm{~A}$ ). Their interaction was confirmed with both full-length sequences. Further, BILBO2-NTD and FPC4-B1BD domains are both necessary and sufficient for the interaction. This was also supported by the proximity of FPC4 and BILBO2 in T. brucei immuno-fluorescence labelling at different stages of the cell cycle (Fig 6B), as it was observed for BILBO1 and FPC4 [15]. Because triple labelling of BILBO1, BILBO2 and FPC4 was challenging, probably due to primary and secondary antibodies steric hindrance, with turned to ultrastructure expansion microscopy (U-ExM) that allows the expansion of a sample and the visualization of preserved ultrastructure of macromolecules by optical microscopy $[22,23]$. This approach facilitated the localization of the FPC (labelled with BILBO1) in respect to the MTQ and to the axoneme that goes through (S6 Fig. A). The MTQ extends from between the mature and the immature basal bodies, turns around the axoneme and is prolonged along the FAZ as previously described in $[5,6]$. Higher resolution of the BILBO1 and BILBO2 colocalization was further determined using the same approach coupled to confocal microscopy (S6 Fig. B and S7 Fig. movie). These approaches allowed us to evidence the annular shape of the FPC and the overall co-localization of BILBO1 and BILBO2 at the FPC. Further, triple labelling of BILBO1, BILBO2 and FPC4 showed that FPC4 partially colocalizes at the FPC and extends on the shank of the Hook Complex with a regular pattern as previously demonstrated in [15] (Fig. $6 \mathrm{C})$. Interestingly, BILBO2 colocalizes with BILBO1 at the FPC following also a regular pattern that extends past the FPC and colocalizes with FPC4.

We then took advantage of the property of FPC4 to bind to MT [15] to assess the localization of ${ }_{\mathrm{HA}} \mathrm{BILBO} 2$ in detergent-extracted U-2 OS cells expressing FPC4 ${ }_{\text {GFP }}$ (Fig 6D). When co-expressed, BILBO2 localized onto the MT labelling of FPC4, confirming their specific interaction in vivo. The deletion of the FPC4-B1BD abolished the interaction and resulted in the removal of BILBO2 during detergent extraction. Interestingly, BILBO2-T2 (aa1-151) can bind to FPC4, whereas BILBO2-T1 (aa 1-103) was extracted, suggesting that this construct might be too short for correct folding or disrupts the binding site for FPC4. Finally, the BILBO2B1BD does not bind to FPC4 and is removed during extraction.

Because BILBO2 binds to BILBO1 (via its CTD) and to FPC4 (via its NTD), we tested whether a tripartite interaction could occur in vivo (Fig 6E). Immuno-labelling of co-expressed BILBO1, 
${ }_{\mathrm{HA}} \mathrm{BILBO} 2$, and $\mathrm{FPC}_{\mathrm{TY} 1}$ in U-2 OS cells demonstrated that both FPC4 and BILBO2 can bind to the BILBO1 polymers (Fig 6E a). This triple co-localization was also observed when BILBO2 deleted for its BILBO1 binding domain (BILBO2-T2, which binds to FPC4 but not to BILBO1) was expressed (Fig 6E b) suggesting a tripartite interaction schematized in Fig 6F.

\section{Crystal structure of the BILBO2-NTD/FPC4-CTD complex provides the first atomic view of two FPC components}

To reveal the molecular mechanisms underlying BILBO2-FPC4 interaction, we have determined a crystal structure of the BILBO2-NTD/FPC4-B1BD complex (Fig 7). The crystal structure was determined at $1.86 \AA$ \& resolution by molecular replacement using the structure of BILBO1-NTD (6JSQ.pdb) as the search model (Table 1). There are two copies of the heterodimer per asymmetric unit in the crystal. Both structures contain aa4-110 of BILBO2 and aa432-438 of FPC4, except aa26-27 that were missing in one BILBO2 molecule. The two structures of the complex are nearly identical, with a root-mean-square deviation (RMSD) of $0.46 \AA$ for all aligned backbone atoms (Fig 7A). These two structures of BILBO2-NTD are also very similar to our previously reported crystal structure of BILBO1-NTD [14], with RMSD values of $0.84 \AA$ and $0.98 \AA$. Similar to the BILBO1-NTD structure, the C-terminal part of BILBO2-NTD (aa91-110) also forms a horseshoe-like loop that wraps around the first two $\beta$ strands and the long $\alpha$ helix of the core structure (Fig 7B). FPC4 is docked into the hydrophobic cleft between the C-terminal loop and the $\beta$ sheet (Fig 7C). Interestingly, despite the fact that we used aa394444 of FPC4 to prepare the sample for crystallization, only residues L432-P438 were visible in the final structure, suggesting that the rest of FPC4 does not bind to BILBO2 and is thus disordered as predicted (Fig 7D). This part of FPC4, particularly residues S435 and P436 in the centre of the stretch, makes extensive contacts with BILBO2 through multiple hydrogen bonds and many hydrophobic interactions (S8 Fig). This structure represents the first-ever highresolution view of intermolecular interactions between two FPC components.

\section{Mutation of critical interface residues abolishes the BILBO2-FPC4 interaction}

A close look in the complex structure suggests that residues S435, P436, and P438 of FPC4 are in close contact with BILBO2 (Fig 8A). We thus mutated each of these three residues to alanine and checked whether the mutants affect the interaction. Firstly, isothermal titration 
calorimetry (ITC) experiments with BILBO2-NTD and FPC4-B1BD demonstrated interaction with a dissociation constant (Kd) of $2.42 \mu \mathrm{M}$ (Fig 8B), which suggested a slightly tighter interaction than that between BILBO1-NTD and FPC4 $(\mathrm{Kd}=5.4 \mu \mathrm{M})$ [15]. For the three mutants, two of them (i.e. S435A and P436A) completely abolished the BILBO2-NTD/FPC4-B1BD interaction, whilst mutant P438A only marginally affected the interaction ( $\mathrm{Kd}=4.31 \mu \mathrm{M})$. The role of S435 and P436 in the interaction was additionally confirmed in the U-2 OS system. BILBO2, which normally binds to FPC4 in these cells (Fig 6C), was detergent-extracted from FPC4-S435A $\mathrm{A}_{\text {GFP }}$ and FPC4-P436A $\mathrm{A}_{\text {GP }}$ expressing cells demonstrating the lack of interaction (Fig $8 \mathrm{C}$, a).

We have previously identified several key aromatic residues in BILBO1-NTD that are important for the BILBO1 function and involved in the FPC4-BILBO1 interaction (residues Y89, F89, W71, Y64) $[14,15]$. The crystal structure of the BILBO2-FPC4 protein complex also suggests that residues F63, F88, and W70 in the BILBO2-NTD are involved in the interaction with FPC4. Here we show in U-2 OS cells that substituting residues F63, F88, or W70 in BILBO2 to alanine indeed abolished the interaction in vivo (Fig 8C,b).

Taken together, these results demonstrate that BILBO2-NTD is homologous to the BILBO1NTD, both structurally and functionally, and that BILBO1, BILBO2, and FPC4 can form a tripartite protein complex that may play an important role in the biogenesis or/and the function of the FPC.

\section{Discussion}

The FPC is a kinetoplastid-specific cytoskeleton structure at the neck of the FP. Although the FPC is essential and required for the biogenesis of the FP and cell survival, its molecular composition and function are poorly known. We previously provided the first molecular evidence that FP biogenesis is mediated by the cytoskeleton via the FPC protein BILBO1 (4). Based on previous work on the polymerization properties of BILBO1 and its interaction with FPC4, a microtubule-binding protein, we hypothesized that BILBO1 forms, at the FPC, a molecular framework upon which other proteins can dock, and that the BILBO1-NTD and EFhands domains could be anchoring or modulator sites $[9-12,15]$. Here, we identify BILBO2 (Tb927.6.3240) as a novel FPC component and as a BILBO1 partner protein. We show that BILBO2 interacts with the BILBO1 EF-hand domains via its C-terminal domain and with FPC4 
via its N-terminal domain. Together with BILBO1, BILBO2 is the second bona fide FPC protein and is involved in a tripartite interaction with BILBO1 and FPC4 (Fig. 6F).

RNAi knockdown of BILBO2 was not lethal, but it is likely that knockdown was incomplete, a phenomenon that has been described before [24]. Repeated failure in producing BILBO2 double knockout in PCF and BSF cell lines supports the essentiality hypothesis and this will be further characterized.

\section{Role of BILBO2 and BILBO1 interaction}

$\mathrm{Y} 2 \mathrm{H}$ assays previously showed that FPC5, a putative kinesin, binds to the BILBO1 EF-hands [10]. Here, we showed that BILBO2 also binds to the BILBO1 EF-hands (Fig 1), but the interaction is not affected by mutating the calcium-binding sites (Fig 2). Moreover, the binding of BILBO2 modulates the shape of the BILBO1 polymer (evidenced from compact to less dense filaments formed by BILBO1 with mutated EF-hands) suggesting that BILBO2 may participate to the plasticity of the BILBO1 polymers. This could be a role for BILBO2 during the cell-cycle. Indeed, BILBO2 FPC-associated protein levels drop by $50 \%$ in $1 \mathrm{~K} 1 \mathrm{~N}-2 \mathrm{FPC}$ cells compared to the $1 \mathrm{~K} 1 \mathrm{~N}-1 \mathrm{FPC}$ levels while BILBO1 level in each FPC remains constant (Fig 3). Cell cycle regulation of BILBO2 was previously demonstrated by the study of the PCF cell cycle proteome where BILBO2 level is maximum in late G1 cells and decreases to $30 \%$ in G2/M cells, and BILBO1 level remains constant [25]. Variation of the BILBO2 levels at the FPC could thus influence the formation or the shape of the BILBO1 polymer and participate in the function or in the biogenesis of the FPC during the cell cycle. Further studies of the BILBO1 polymer structure in the absence and in presence of BILBO2 will provide important clues to understand the role of the FPC architecture.

STED microscopy previously showed a dotted pattern parallel to the FPC and along the MTQ on the distal side of the FPC for FPC4 [15]. Interestingly, BILBO2 is also observed as dotted pattern along the MTQ but also at the FPC. Three dimensional rendering of the expansion microscopy labelling (S6 Fig. B) indicated that BILBO1 and BILBO2 do not localise to the same microtubules in the distal part of the MTQ. The reason for these different localizations is unknow and will be further studied. Fine localization of BILBO1 and BILBO2 at the FPC and the MTQ is currently under investigation to further understand their relationship. 


\section{Crystal structure of the BILBO2-FPC4 complex}

In $\mathrm{Y} 2 \mathrm{H}$ assays and U-2 OS cells, the BILBO2 T2 construct (aa1-151) interacts with FPC4 whilst T1 (aa1-103) did not, suggesting that aa104-151 are involved in the interaction. The crystal structure of the BILBO2-NTD (aa1-110) in complex with the FPC4-B1BD (aa354-404) reveals that the aa91-110 form an extended loop wrapping around the first two $\beta$ strands and the long $\alpha$ helix of the core structure, which helps the protein to form a horseshoe-like hydrophobic pocket for FPC4 binding. This is reminiscent of what was reported for BILBO1NTD [14]. Thus, the T1 construct (aa1-103) is too short to form the intact binding site for FPC4.

Interestingly, only five residues (aa432-438) of the FPC4-B1BD (aa354-444) are visible in the crystal structure of the protein complex. The stretch of FPC4 binds to the hydrophobic pocket and passes through the gap of the horseshoe gauged by the highly conserved aromatic residue W70. The interaction is confirmed by the abolished interaction upon mutating W70, as well as the conserved aromatic residue F88 in the centre of the pocket, to alanine. Conversely, mutation to alanine of the two central residues S435/P436 of the bound peptide completely disrupts the interaction of FPC4 with BILBO2. This is similar to the reported interaction between FPC4 and BILBO1 [15] and suggests that FPC4 might use a similar strategy to bind BILBO1 and BILBO2. Notably, the binding affinity between FPC4 and BILBO2 (Kd = 2.4 $\mu \mathrm{M})$ is higher than that between FPC4 and BILBO1 $(\mathrm{Kd}=\sim 6 \mu \mathrm{M})[15]$. Such a subtle difference in binding affinities suggests possible hierarchical orders in recognizing the two partner proteins in the parasite.

\section{The BILBO1-BILBO2-FPC4 tripartite interaction}

We have demonstrated that BILBO2 in T. brucei is localized at the FPC, is a novel BILBO1 binding protein, and that it is involved in a tripartite interaction with BILBO1 and FPC4 (schematized in Fig 6F). The tripartite interacting domains have been identified, and mutational analyses in U-2 OS cells suggest that BILBO2 can modulate the shape of the BILBO1 filaments by interacting with the BILBO1 EF-hand domains.

Further, we demonstrate that BILBO1 and BILBO2 share a structurally and functionally similar NTD domain with similar key residues interacting with FPC4. BILBO2-NTD and FPC4 interact in $\mathrm{Y} 2 \mathrm{H}$ experiments, in U-2 OS cells, and in vitro, but the interaction is very weak in $T$. brucei detergent-extracted cytoskeleton (Fig 3D). The same is true for the BILBO1-NTD 
constructs and FPC4-B1BD constructs that were not observed at the FPC after detergentextraction in trypanosomes $[10,15]$. This suggests either low-affinity interaction in vivo, or that the tripartite BILBO1-BILBO2-FPC4 interaction is necessary to dock FPC4 and BILBO2 at the FPC. However, FPC4 localizes at the edge of the FPC and HC structures [15] whilst BILBO2 colocalizes with BILBO1 on the whole FPC structure. This might suggest that BILBO2 interacts with FPC4 on the edge of the FPC and along the MTQ.

The BILBO1-BILBO2-FPC4 tripartite interaction might occur exclusively at the proximal portion of the FPC indicating that other, yet to be discovered, proteins are likely to be involved in this protein complex. It is worth mentioning that the binding affinity of FPC4 to BILBO2 is higher than with BILBO1 [15]. We, therefore, speculate that a hierarchy exists where BILBO1 may allow FPC4 to come into close proximity to BILBO2 and then FPC4 interacts with BILBO2. Notably, BILBO2 also interacts with BILBO2 in $\mathrm{Y} 2 \mathrm{H}$ assays suggesting some degree of dimerization or polymerization can occur.

\section{Conclusion}

BILBO1, BILBO2 and FPC4 clearly interact to potentially form a tripartite complex in vivo, and the NTDs of the former two play a significant role in their interaction. It would be important to further understand the role of these NTDs by searching for potential new partners. Overall, our structural and functional data have shed light on the first structure of an FPC protein complex and represent an additional step in deciphering the FPC structure and function in T. brucei. 


\section{Materiel and methods}

\section{Cell lines, culture and transfection}

Trypanosoma. The trypanosome cell lines used in this study were derived from the parental (WT) procyclic form SmOxPCF427 (PCF) and bloodstream form SmOxBSF427 (BSF), both coexpressing the T7 RNA polymerase and tetracycline repressor [26]. PCF cells were cultured at $27^{\circ} \mathrm{C}$ in SDM79 medium (PAA, Ref : G3344,3005) containing $10 \%(\mathrm{v} / \mathrm{v}$ ) heat-inactivated foetal calf serum, $10 \mu \mathrm{g} \cdot \mathrm{ml}^{-1}$ Hemin, $26 \mu \mathrm{M}$ sodium bicarbonate, $10 \mathrm{mM}$ D-Glucose, $3.5 \mathrm{mM} \mathrm{L-}$ glutamine, $5.3 \mathrm{mM}$ L-proline, $0.9 \mathrm{mM}$ sodium pyruvate, $3.4 \mathrm{mM}$ L-threonine, $150 \mu \mathrm{M}$ glutamic acid, $120 \mu \mathrm{M}$ sodium acetate, $230 \mu \mathrm{M}$ D-glucosamine, puromycin $1 \mu \mathrm{g} \cdot \mathrm{ml}^{-1}$. BSF cells were cultured at $37^{\circ} \mathrm{C}$ and $5 \% \mathrm{CO}_{2}$ as described in [19] in IMDM medium containing $10 \%(\mathrm{v} / \mathrm{v}$ ) heatinactivated foetal calf serum, $36 \mathrm{mM}$ sodium bicarbonate, $136 \mu$ g.mL-1 hypoxanthine, $39 \mu$ g. $\mathrm{mL}^{-1}$ thymidine, $110 \mu \mathrm{g} \cdot \mathrm{mL}^{-1}$ sodium pyruvate, $28 \mu \mathrm{g} \cdot \mathrm{mL}-1$ bathocuproine, $0.25 \mathrm{mM} \beta$ mercaptoethanol, $2 \mathrm{mM}$ L-cysteine, $62.5 \mu \mathrm{g} \cdot \mathrm{mL}^{-1}$ kanamycin, $0.1 \mu \mathrm{g} \cdot \mathrm{mL}^{-1}$ puromycin, hygromycin $25 \mu \mathrm{g} \cdot \mathrm{mL}^{-1}$ or $5 \mu \mathrm{g} \cdot \mathrm{mL}^{-1}$. Cells were transfected as described in [27] using an AMAXA electroporator and program X-001 with the transfection buffer described in [28]. After transfection, the cells were selected using the appropriate antibiotic (for PCF and BSF respectively blasticidin $20 \mu \mathrm{g} \cdot \mathrm{mL}^{-1}$ or $10 \mu \mathrm{g} \cdot \mathrm{mL}^{-1}$, phleomycin $5 \mu \mathrm{g} \cdot \mathrm{mL}^{-1}$ or $2.5 \mu \mathrm{g} \cdot \mathrm{mL}^{-1}$, neomycin $10 \mu \mathrm{g} \cdot \mathrm{mL}^{-1}$ or $\left.2.5 \mu \mathrm{g} \cdot \mathrm{mL}^{-1}\right)$. Clones were selected after serial dilutions. Ectopic expression and RNAi were induced with tetracycline at $1 \mu \mathrm{g} \cdot \mathrm{mL}^{-1}$ and $10 \mu \mathrm{g} \cdot \mathrm{mL}^{-1}$, respectively.

U-2 OS cells (human bone osteosarcoma epithelial cells, ATCC Number: HTB-96 [29] were grown in D-MEM Glutamax (Gibco) supplemented with $10 \%$ foetal calf serum and $1 \%$ penicillin-streptomycin at $37^{\circ} \mathrm{C}$ plus $5 \% \mathrm{CO}_{2}$. Exponentially growing cells were transfected with 0.5-1 $\mu \mathrm{g}$ DNA using Lipofectamine 2000 in OPTIMEM (Invitrogen) according to the manufacturer's instructions and processed for IF $24 \mathrm{~h}$ post-transfection.

\section{Molecular biology, cloning}

BILBO2 ORF was initially cloned by PCR using Tb927 genomic DNA as a template.

Trypanosome vectors. For the RNAi experiments in PCF and BSF, the BILBO2 fragment (bp 205-816) was cloned between the $\mathrm{Xbal} / \mathrm{Xhol}$ restriction sites of the p2T7-177 vector [30]. The full-length BILBO2 ORF and truncations were cloned into the HinIII/Xhol sites of pFS-1 vector 
containing a C-terminal 3HA-tag to express BILBO2-HA, BILBO2-T1-HA, BILBO2-T2-HA and BILBO2$\mathrm{B} 1 \mathrm{BD}_{-\mathrm{HA}}$ (vector modified from [31]). For N-terminal 10xTY1 tagging of BILBO2, long primers were designed as described in [19] and the PCR was performed with the pPOTv7 vector template. The PCR product was transfected as described in [19].

Yeast-two hybrid vectors. Genes encoding proteins and protein domains were amplified and cloned into the Ndel-BamHI sites (for BILBO2) of the pGADT7 and pGBKT7 plasmids (Clontech). The TbBILBO1 and TbFPC4 vectors are described in $[10,15]$.

Mammalian vectors. The BILBO1 ORF was cloned into the pcDNA3 vector as described in [10]. The BILBO2 ORF or truncations were cloned into the pcDNA3.1 vector, in frame with Nterminal 3xHA tag, from which the mutants for serine 171 and 173 were generated using the QuickChange II site-directed mutagenesis kit (Agilent). The BILBO1, BILBO1 $1_{\text {GFP }}$, FPC4 $_{\text {GFP }}$ FPC4$B 1 B D_{G F P}$ and FPC4- $\triangle B 1 B D_{\text {GFP }}$ constructs are described in $[10,15]$. The FPC4 ORF was also cloned into the pcDNA3.1 vector, in frame with a C-terminal 3xTY1 tag.

Bacterial vectors. To allow the coexpression of BILBO2-NTD and FPC4-B1BD, two vectors containing different antibiotic resistances were used. Both vectors contain a similar $\mathrm{His}_{6}{ }^{-}$ SUMO tag, which was included to increase protein expression and solubility of recombinant proteins $[33,34]$. The constructs also contain a cleavage site between the tag and the target proteins, which is cut by the highly specific SENP2 (Sentrin/SUMO-specific protease 2) protease. The SUMOpET15b vector, containing ampicillin resistance and $\mathrm{Ndel} / \mathrm{BamHI}$ cleavage sites, was used to express BILBO2-NTD. The SUMOpET28a vector, containing kanamycin resistance and BamHI/Xhol cleavage sites, was used to express wild-type and mutants of FPC4B1BD. All mutants were generated by site-directed mutagenesis using a QuikChange kit (Stratagene) according to the manufacturer's instructions. Incorporations of mutations were confirmed by DNA sequencing.

Yeast vectors. Sequences encoding BILBO2 and truncations were cloned into PGBKT7 and sequences encoding BILBO1 and truncations were cloned into pGADT7 (Clontech).

\section{Production of guinea pig anti-BILBO2 serum.}


BILBO2 ORF was cloned into pET28a(+) (NOVAGEN) and expressed in BL21(DE3) E. coli, and purified in $8 \mathrm{M}$ urea, $50 \mathrm{mM}$ NaPi buffer $\mathrm{pH} 7.4,500 \mathrm{mM} \mathrm{NaCl}$. Guinea pig immunization was done by Eurogentec.

\section{Immunofluorescence}

Wide-field fluorescence microscopy on trypanosome. Cells were processed and fixed as described in [10], except for BSF that were washed in vPBS ( $\mathrm{NaCl} 0.8 \mathrm{mg} \cdot \mathrm{mL}^{-1}, \mathrm{KCl} 0.22 \mathrm{mg} \cdot \mathrm{mL}^{-}$ 1, $\mathrm{Na}_{2} \mathrm{HPO}_{4} 22.7$ g.mL $\mathrm{mL}^{-1}, \mathrm{KH}_{2} \mathrm{PO}_{4} 4.4 \mathrm{mg} \cdot \mathrm{mL}^{-1}$, sucrose $15.7 \mathrm{mg} \cdot \mathrm{mL}^{-1}$, glucose $1.8 \mathrm{mg} \cdot \mathrm{mL}^{-1}$ ), resuspended in $0.25 \%$ Nonidet P-40 (IGEPAL), $100 \mathrm{mM}$ PIPES-NaOH pH6.9, $1 \mathrm{mM} \mathrm{MgCl}$, and loaded on poly-L-lysine-coated slides for $10 \mathrm{~min}$. After either $3 \%$ paraformaldehyde (PFA) fixation for $5 \mathrm{~min}$ at RT (then $10 \mathrm{mM}$ glycine neutralization) or $-20^{\circ} \mathrm{C}$ methanol fixation for 30 min, the slides were washed in PBS twice for 5 min and incubated with primary antibodies in PBS for $1 \mathrm{~h}$ in a dark moist chamber (anti-BILBO1 1-110 [10], 1:4,000 dilution; anti-HA tag IgG1 mouse monoclonal, Biolegend 901514, 1:1000; IgG2a mouse monoclonal, GeneTex GTX628902, 1:2000; mouse anti-TY1 tag BB2 IgG1, 1:1000 [35]). Following the primary antibody incubation, samples were washed twice $(5 \mathrm{~min}$ ) in PBS and incubated $1 \mathrm{~h}$ with the secondary antibodies [anti-rabbit IgG conjugated to FITC (Sigma F-2012, 1:100); anti-rabbit IgG conjugated to Alexa fluor 594 (Molecular Probes A-11012, 1:100); anti-mouse IgG1 conjugated to Alexa fluor 594 (Molecular Probes A-21125, 1:100); anti-mouse IgG2a conjugated to Alexa fluor 488 (Molecular Probes A-21131, 1:100); anti-rabbit conjugated to Alexa fluor 647 (Molecular Probes A-31573, 1:100)] according to the primary antibody combination. After two 5 min washes in PBS, kinetoplasts and nuclei were labelled for 5 min with DAPI $\left(10 \mu \mathrm{g} \cdot \mathrm{mL}^{-1}\right)$ followed by two PBS washes. Slides were mounted with SlowFade ${ }^{\circledR}$ Gold Kit (Molecular Probes, S-36936).

Wide-field fluorescence microscopy on U-2 OS cells. U-2 OS cells grown on glass coverslips were washed with $\mathrm{PBS}$, fixed in $3 \%$ paraformaldehyde for 15 minutes (at $37^{\circ} \mathrm{C}$ ) and permeabilized 30 min in PBS containing 10\% FCS and $0.1 \%$ saponin or briefly extracted with an extraction buffer (0.5\% TX-100, 10\% glycerol in EMT [60 mM PIPES-NaOH pH6.9, 25 mM HEPES, $\left.10 \mathrm{mM} \mathrm{EGTA,} 10 \mathrm{mM} \mathrm{MgCl}_{2}\right]$ ) to obtain cytoskeletons and fixed in $3 \%$ paraformaldehyde in $\mathrm{PBS}$ for 15 minutes (at $37^{\circ} \mathrm{C}$ ). Samples were then processed for 
immunofluorescence as in [10]. The primary antibodies (anti-BILBO1 1-110, 1:4000 dilution; anti-living colours rabbit polyclonal Clontech, 1:1,000 dilution; anti-HA tag mouse monoclonal IgG1 Biolegend, 1:1,000 dilution) were incubated for $1 \mathrm{~h}$ in a dark moist chamber. After two PBS washes, cells were incubated for $1 \mathrm{~h}$ with the secondary antibodies anti-rabbit IgG conjugated to Alexa fluor 594 (Molecular Probes, 1:400); anti-mouse IgG conjugated to FITC (Sigma, 1:400). The nuclei were stained with DAPI (0.20 $\mu \mathrm{g} \cdot \mathrm{mL}^{-1}$ in PBS for $\left.5 \mathrm{~min}\right)$, then washed twice in PBS and mounted overnight with Prolong (Molecular Probes S-36930). Images were acquired on a Zeiss Imager Z1 microscope with Zeiss 100x or 63x objectives (NA 1.4), using a Photometrics Coolsnap HQ2 camera and Metamorph software (Molecular Devices), and processed with ImageJ.

Ultrastructure Expansion Microscopy. The protocol was adapted from [23,36]. T. brucei PCF cells expressing endogenously ${ }_{10 m y c} \mathrm{BILBO} 2$ and ${ }_{10 \mathrm{TY} 1 \mathrm{FPC}}$ (500 $\mu$ l of culture corresponding to 4.106 cells per coverslip) were loaded on poly-L-lysine coated 12-mm coverslips in 24-well plate and cells left to adhere between 5 to 10 minutes and extracted as described above. Cytoskeletons were covered with $1 \mathrm{~mL}$ of activation solution $(0.7 \%$ formaldehyde; $1 \%$ Acrylamide in PBS ) for 4 hours at $37^{\circ} \mathrm{C}$ with slow agitation. For the gelation step, coverslips were gently deposited on top of a $35 \mu$ drop of MS solution (23\% Sodium acrylate (Sigma 408220); 10\% Acrylamide (Euromedex EU0060-A); 0.1\% Bis-acrylamide (Euromedex EU0560A) in PBS) for 2 min then transferred to a $35 \mu$ drop of MS; 0.5\% TEMED (Euromedex 50406A); $0.5 \%$ Ammonium persulfate (Euromedex EU0009-A) for 5 minutes on ice then transferred at $37^{\circ} \mathrm{C}$ and incubated for 1 hour without agitation. The coverslips were then transferred in 6well plate in $1 \mathrm{~mL}$ of denaturation solution (200 mM Sodium Dodecyl Sulfate; $200 \mathrm{mM}$ Sodium chloride; $50 \mathrm{mM}$ Tris $\mathrm{pH}$ 9.0) with agitation at RT for $15 \mathrm{~min}$ to detach the gel from the coverslip, then moved into a $1.5 \mathrm{ml}$ Eppendorf centrifuge tube filled with denaturation solution and incubated at $95^{\circ} \mathrm{C}$ for one 90 minutes. Gels were expanded in large volumes of deionized water (twice 30 min then overnight) then incubated in a large volume of PBS for 10 minutes (three times). Small pieces of the gels were processed for immuno-labelling as follows. The gels were preincubated in blocking solution (PBS, 2\% BSA, 0.2\% Tween-20) for 30 $\min \left(37^{\circ} \mathrm{C}\right)$. The primary antibodies (rabbit anti-BILBO1 1-110, 1:1000 dilution; mouse IgG1 anti c-Myc clone 9E10, 1:500 dilution; mouse IgG2a anti c-Myc clone 9B11 (Cell Signalling), 
1:1000 dilution; mouse anti-TY1 (BB2) [35], 1:1000 dilution, mouse IgG1 anti-Tubulin (DM1A Sigma) 1:250; mouse IgG2a anti-TbSAXO mAb25 [37] 1:5) diluted in blocking solution were incubated for 3 hours in the dark at $37^{\circ} \mathrm{C}$ with slow agitation. After three washes in blocking solution, gels were incubated with the secondary antibodies (anti-Rabbit Alexa fluor594 conjugated (Molecular Probes, 1:500 dilution); anti-Mouse Alexa fluor conjugated 488 (Molecular Probes, 1:200 dilution; anti-Rabbit Alexa fluor 647 conjugated (Molecular Probes, 1:200 dilution); anti-Mouse IgG1 specific Alexa fluor 594 conjugated (Molecular Probes, 1:500 dilution); anti-Mouse IgG2a specific Alexa fluor 488 (Molecular Probes, 1:1000 dilution)) diluted in blocking solution for 3 hours in the dark at $37^{\circ} \mathrm{C}$ with slow agitation. After three washes in blocking solution, gels were expanded a large volume of deionized water (twice 30 minutes then overnight). The expansion factor was determined using the ratio between the size of the coverslip $(12 \mathrm{~mm})$ and the size of the gels after the first expansion. Images were acquired on a Zeiss Imager Z1 microscope with Zeiss 63x oil objectives (NA 1.4), using a Photometrics Coolsnap HQ2 camera and Metamorph software (Molecular Devices), and processed with ImageJ. U-ExM images were acquired on a Zeiss Imager Z1 microscope (Fig. 6C and S6 Fig. A) and a Leica SP8 WLL2 on an inverted stand DMI6000 (Leica Microsystems, Mannheim, Germany), using a 63X oil objective (HCX Plan Apo CS2, NA 1.40) (S6 Fig. B and S7 Fig. movie) and processed with FIJI.

\section{Expression and purification of BILBO2-NTD and FPC4-B1BD}

Recombinant BILBO2-NTD and FPC4-B1BD proteins were expressed in E. coli (strain BL21DE3). Expression and purification of single proteins were carried out in a similar way to the coexpression described below. For co-expression, the two cloned constructs were cotransformed into competent bacterial cells. The cells were grown in Luria-Bertani (LB) medium at $37{ }^{\circ} \mathrm{C}$ to an $\mathrm{OD}_{600}$ of $\sim 0.6$ and then subjected to cold shock on ice for 20 min. Protein expression was induced by addition of $0.5 \mathrm{mM}$ isopropyl $\beta$-D-thiogalactopyranoside, and cell cultures were further incubated at $16{ }^{\circ} \mathrm{C}$ overnight $(\sim 16 \mathrm{~h})$. Cells were harvested by centrifugation in a Sorvall GS3 rotor $\left(6,000 \times \mathrm{g}, 12 \mathrm{~min}, 4^{\circ} \mathrm{C}\right)$ and then resuspended in the lysis buffer containing $20 \mathrm{mM}$ Tris- $\mathrm{HCl}, \mathrm{pH} 8.0,100 \mathrm{mM} \mathrm{NaCl}, 20 \mathrm{mM}$ imidazole, $10 \mathrm{mM} \beta$ mercaptoethanol, and 5\% (v/v) glycerol (20 ml buffer per litre of cell culture). Cells were lysed in an Emulsiflex C3 homogenizer (Avestin) and cell debris was pelleted by centrifugation 
$\left(40,000 \times \mathrm{g}, 30 \mathrm{~min}, 4^{\circ} \mathrm{C}\right)$. The supernatant was filtered $(0.45-\mu \mathrm{m}$ pore size, Amicon) and loaded onto a Ni-HiTrap column (GE Healthcare) which was pre-equilibrated with the same lysis buffer. The column was washed with $5 \times$ column volume (CV) of lysis buffer, and bound protein was eluted using a linear gradient concentration of imidazole $(20-500 \mathrm{mM}, 20 \times \mathrm{CV})$. The $\mathrm{His}_{6}$-SUMO tag on both proteins was of TbBILBO1-NTD was cleaved off by incubating the eluted proteins with $\sim 1 \%(\mathrm{w} / \mathrm{w})$ of SENP2 $\left(4{ }^{\circ} \mathrm{C}\right.$, overnight). Target proteins were further purified on a 16/60 Superdex-200 column (GE Healthcare) pre-equilibrated with a running buffer containing $20 \mathrm{mM}$ Tris- $\mathrm{HCl}(\mathrm{pH} 8.0)$ and $100 \mathrm{mM} \mathrm{NaCl}$. Elution peaks containing both proteins were pooled and concentrated to $\sim 20 \mathrm{mg} / \mathrm{ml}$ for crystallization trials.

\section{Crystallization and structure determination}

Purified protein of the BILBO2-NTD/FPC4-B1BD complex was used to set up crystallization trials. Initial conditions giving small seed-like crystals were further optimized. Single diamondlike crystals with dimensions of approximately $100 \times 50 \times 50 \mu \mathrm{m}$ were obtained under a condition containing $0.1 \mathrm{mM} \mathrm{NaOAc}(\mathrm{pH} 4.5), 0.5 \mathrm{M}$ 1,6-Hexanediol, and $10 \mathrm{mM} \mathrm{CoCl}_{2}$.

Crystals were harvested by sequentially soaked in the same crystallization solution with increasing concentration of glycerol [5-20\% (v/v)], mounted onto nylon loops, and then flashfrozen in liquid nitrogen. Data collection was carried out at the beamline ID23-1 of the European Synchrotron Radiation Facility (ESRF) at the wavelength of $0.9763 \AA$. Diffraction data were processed by the XDS program [38]. Structure determination was carried out using the molecular replacement method by the program Phaser within the Phenix suite [39]. Partially built models were checked and missing loops manually added in COOT [40]. Structure refinement was carried out using the phenix.refine [41].

\section{Yeast two-hybrid assay}

Interactions assays were done on SC-W-L-H medium as described in [10]. Photos were acquired after 3 days of incubation.

\section{Western-blotting}

Sample preparation for whole cells and cytoskeleton. $2.10^{7}$ cells were split for whole cells (WC) and cytoskeleton (CSK) samples. For WC samples, cells were spun at 1,000 x g for 10 minutes, 
washed once and resuspended at $1.10^{6}$ cells/ $\mu \mathrm{L}$ in PBS. An equivalent volume of $2 x$ sample buffer and $25 \mathrm{U}$ of benzonase (Sigma, E1014) was added before boiling 5 minutes. For CSK samples, cells were spun at 1,000 g for 10 minutes and washed once in PBS, EDTA 10mM and resuspended at $1.10^{6}$ cells/ $\mu \mathrm{L}-1$ in $100 \mathrm{mM}$ PIPES pH6.8, $2 \mathrm{mM} \mathrm{MgCl}_{2}, 0.25 \%$ NP-40 (Igepal Sigma), Protease inhibitor (Calbiochem, 1:10,000 dilution) and 25U of benzonase. After 10 minutes incubation on ice, cytoskeletons were pelleted at 1,000 $\mathrm{g}$ for 30 minutes then washed in $1 \mathrm{~mL} 100 \mathrm{mM}$ PIPES pH6.8, $2 \mathrm{mM} \mathrm{MgCl}_{2}$ and resuspended in the same buffer $\left(1.10^{6}\right.$ cells $/ \mu \mathrm{L}$ final). An equivalent volume of $2 \mathrm{x}$ sample buffer was added before boiling for 5 minutes. Protein samples (equivalent to $5.10^{6}$ trypanosomes whole cell or cytoskeletons) were separated on SDS-PAGE gels and transferred by semi-dry (BioRad) blotting $45 \mathrm{~min}$ at $25 \mathrm{~V}$ on PVDF or PVDF low fluorescence PVDF membrane. After a $1 \mathrm{~h}$ blocking step in 5\% skim milk in TBS-0.2\% Tween-20, the membranes were incubated overnight at $4^{\circ} \mathrm{C}$ with the primary antibodies diluted in blocking buffer (anti-enolase (rabbit polyclonal, 1:25,000); anti-tubulin TAT1 (mouse monoclonal, 1:1,000 [42]); anti-BILBO1 1-110 (rabbit polyclonal, 1:1,000 [15]); anti-TY1 BB2 (mouse monoclonal 1:50,000 [35]); anti-HA (Biolegend mouse monoclonal, 1:1,000). After three washes in blocking buffer, the membranes were incubated with the secondary antibodies (anti-mouse HRP-conjugated (Jackson Immunoresearch, 1:10,000); antirabbit HRP-conjugated (Sigma, 1:10,000 dilution)) and washed twice $10 \mathrm{~min}$ in blocking buffer and twice 5 min in PBS. Blots were revealed using the Clarity Western ECL Substrate kit (BioRad) with the ImageQuant LAS4000 (GE Healthcare).

\section{Isothermal titration calorimetry (ITC)}

Purified BILBO2-NTD and FPC4-B1BD (WT or mutated) were dialyzed overnight against a buffer containing $20 \mathrm{mM}$ Tris- $\mathrm{HCl}(\mathrm{pH} 8.0)$ and $50 \mathrm{mM} \mathrm{NaCl}$. Protein concentration was determined by ND-1000 spectrophotometer (PEQlab). ITC experiments were carried out at $25^{\circ} \mathrm{C}$ using an iTC200 microcalorimeter (MicroCal, GE healthcare). The cell contained $200 \mu \mathrm{l}$ of $50 \mu \mathrm{M}$ BILBO2-NTD constructs, which was titrated with an initial $0.4 \mu \mathrm{l}$ injection followed by 19 constitutive injections ( $2 \mu$ leach) of $600 \mu \mathrm{M}$ FPC4-B1BD with a duration of $0.8 \mathrm{~s}$. The interval between every two injections was $150 \mathrm{~s}$. The ITC data were analysed using the program Origin version 7.0 provided by MicroCal. The One-site binding model was used to fit the integrated data to calculate the stoichiometry and binding constants. 
Accession code: Coordinates and structure factors of the crystal structure of the BILBO2NTD/FPC4-CTD complex have been deposited in the Protein Data Bank (PDB) under accession code 7a1i.

\section{Acknowledgements}

We thank Annelise Sahin and Marie Eggenspieler for their help at the beginning of the study. We thank F. Bringaud (University of Bordeaux) for the anti-enolase antibody, K. Gull (Oxford University) for the anti-tubulin TAT1 antibody, P. Bastin (Institut Pasteur) for the anti-Ty1 antibody, Samuel Dean (Warwick Medical School) and Jack Sunter (Oxford Brookes University) for the pPOT plasmids and SmOx T. brucei cell lines. We are grateful to the staff at the beamline of ID23-1 at the European Synchrotron Radiation Facility (ESRF) for their help with X-ray diffraction. This work was supported by the CNRS and the University of Bordeaux to DRR and MB, the LabEx ParaFrap [ANR-11-LABX-0024] to DRR, the Max Perutz Labs and grant [P24383-B21] from the Austrian Science Fund (FWF) to GD, the ANR-FWF PRCI [ANR-20-CE910003; P24383-B21] to MB and GD. Cl was supported by the LabEx Parafrap PhD program [ANR11-LABX-0024], and YP was supported by the "Integrative Structural Biology" PhD program [W-1258 Doktoratskollegs] funded by the FWF. Some of the U-ExM microscopy was done in the Bordeaux Imaging Center a service unit of the CNRS-INSERM and Bordeaux University, member of the national infrastructure France Biolmaging supported by the French National Research Agency [ANR-10-INBS-04]. The help of Magali Mondin is acknowledged. We thank J. Marcos, G. Cougnet-Houlery, and S. Guit for the continued MFP lab infrastructure. 
References

1. Gull K. The cytoskeleton of trypanosomatid parasites. Annu Rev Microbiol. 1999;53: 629-55.

2. Field MC, Carrington M. The trypanosome flagellar pocket. Nat Rev Microbiol. 2009;7: 775-786. doi:10.1038/nrmicro2221

3. Robinson DR, Sherwin T, Ploubidou A, Byard EH, Gull K. Microtubule polarity and dynamics in the control of organelle positioning, segregation, and cytokinesis in the trypanosome cell cycle. J Cell Biol. 1995;128: 1163-1172. doi:10.1083/jcb.128.6.1163

4. Bonhivers M, Nowacki S, Landrein N, Robinson DR. Biogenesis of the Trypanosome Endo-Exocytotic Organelle Is Cytoskeleton Mediated. PLOS Biol. 2008;6: e105. doi:10.1371/journal.pbio.0060105

5. Lacomble S, Vaughan S, Gadelha C, Morphew MK, Shaw MK, McIntosh JR, et al. Three-dimensional cellular architecture of the flagellar pocket and associated cytoskeleton in trypanosomes revealed by electron microscope tomography. J Cell Sci. 2009;122: 108190. doi:10.1242/jcs.045740

6. Esson HJ, Morriswood B, Yavuz S, Vidilaseris K, Dong G, Warren G. Morphology of the Trypanosome Bilobe, a Novel Cytoskeletal Structure. Eukaryot Cell. 2012;11: 761-772. doi:10.1128/EC.05287-11

7. Morriswood B. Form, Fabric, and Function of a Flagellum-Associated Cytoskeletal Structure. Cells. 2015;4: 726-747. doi:10.3390/cells4040726

8. Morriswood B, Schmidt K. A MORN Repeat Protein Facilitates Protein Entry into the Flagellar Pocket of Trypanosoma brucei. Eukaryot Cell. 2015;14: 1081-1093. doi:10.1128/EC.00094-15

9. Vidilaseris K, Shimanovskaya E, Esson HJ, Morriswood B, Dong G. Assembly Mechanism of Trypanosoma brucei BILBO1, a Multidomain Cytoskeletal Protein. J Biol Chem. 2014;289: 23870-23881. doi:10.1074/jbc.M114.554659

10. Florimond C, Sahin A, Vidilaseris K, Dong G, Landrein N, Dacheux D, et al. BILBO1 Is a Scaffold Protein of the Flagellar Pocket Collar in the Pathogen Trypanosoma brucei. PLoS Pathog. 2015;11. doi:10.1371/journal.ppat.1004654

11. Perdomo D, Bonhivers M, Robinson DR. The Trypanosome Flagellar Pocket Collar and Its Ring Forming Protein-TbBILBO1. Cells. 2016;5: 9. doi:10.3390/cells5010009

12. Vidilaseris K, Lesigang J, Morriswood B, Dong G. Assembly mechanism of Trypanosoma brucei BILBO1 at the flagellar pocket collar. Commun Integr Biol. 2015;8. doi:10.4161/19420889.2014.992739

13. Vidilaseris K, Morriswood B, Kontaxis G, Dong G. Structure of the TbBILBO1 protein $\mathrm{N}$-terminal domain from Trypanosoma brucei reveals an essential requirement for a conserved surface patch. J Biol Chem. 2014;289: 3724-35. doi:10.1074/jbc.M113.529032

14. Vidilaseris K, Landrein N, Pivovarova Y, Lesigang J, Aeksiri N, Robinson DR, et al. Crystal structure of the $\mathrm{N}$-terminal domain of the trypanosome flagellar protein BILBO1 reveals a ubiquitin fold with a long structured loop for protein binding. J Biol Chem. 2020;295: 1489-1499. doi:10.1074/jbc.RA119.010768

15. Albisetti A, Florimond C, Landrein N, Vidilaseris K, Eggenspieler M, Lesigang J, et al. Interaction between the flagellar pocket collar and the hook complex via a novel microtubule-binding protein in Trypanosoma brucei. PLOS Pathog. 2017;13: e1006710. doi:10.1371/journal.ppat.1006710 
16. McAllaster MR, Ikeda KN, Lozano-Núñez A, Anrather D, Unterwurzacher V, Gossenreiter $\mathrm{T}$, et al. Proteomic identification of novel cytoskeletal proteins associated with TbPLK, an essential regulator of cell morphogenesis in Trypanosoma brucei. Mol Biol Cell. 2015;26: 3013-3029. doi:10.1091/mbc.E15-04-0219

17. Aslett M, Aurrecoechea C, Berriman M, Brestelli J, Brunk BP, Carrington M, et al. TriTrypDB: a functional genomic resource for the Trypanosomatidae. Nucleic Acids Res. 2010;38: D457-62. doi:10.1093/nar/gkp851

18. Urbaniak MD, Martin DMA, Ferguson MAJ. Global Quantitative SILAC Phosphoproteomics Reveals Differential Phosphorylation Is Widespread between the Procyclic and Bloodstream Form Lifecycle Stages of Trypanosoma brucei. J Proteome Res. 2013;12: 2233. doi:10.1021/pr400086y

19. Dean S, Sunter J, Wheeler RJ, Hodkinson I, Gluenz E, Gull K. A toolkit enabling efficient, scalable and reproducible gene tagging in trypanosomatids. Open Biol. 2015;5: 140197. doi:10.1098/rsob.140197

20. Wirtz E, Leal S, Ochatt C, Cross GA. A tightly regulated inducible expression system for conditional gene knock-outs and dominant-negative genetics in Trypanosoma brucei. Mol Biochem Parasitol. 1999;99: 89-101.

21. Alsford S, Turner DJ, Obado SO, Sanchez-Flores A, Glover L, Berriman M, et al. Highthroughput phenotyping using parallel sequencing of RNA interference targets in the African trypanosome. Genome Res. 2011;21: 915-924. doi:10.1101/gr.115089.110

22. Gambarotto D, Zwettler FU, Guennec ML, Schmidt-Cernohorska M, Fortun D, Borgers $\mathrm{S}$, et al. Imaging cellular ultrastructures using expansion microscopy (U-ExM). Nat Methods. 2019;16: 71-74. doi:10.1038/s41592-018-0238-1

23. Amodeo S, Kalichava A, Fradera-Sola A, Bertiaux-Lequoy E, Guichard P, Butter F, et al. Characterization of the Novel Mitochondrial Genome Segregation Factor TAP110 in Trypanosoma brucei. bioRxiv. 2020; 2020.06.25.171090. doi:10.1101/2020.06.25.171090 24. Wang Z, Morris JC, Drew ME, Englund PT. Inhibition of Trypanosoma brucei Gene Expression by RNA Interference Using an Integratable Vector with Opposing T7 Promoters. J Biol Chem. 2000;275: 40174-40179. doi:10.1074/jbc.M008405200

25. Crozier TWM, Tinti M, Wheeler RJ, Ly T, Ferguson MAJ, Lamond Al. Proteomic Analysis of the Cell Cycle of Procylic Form Trypanosoma brucei. Mol Cell Proteomics MCP. 2018;17: 1184-1195. doi:10.1074/mcp.RA118.000650

26. Poon SK, Peacock L, Gibson W, Gull K, Kelly S. A modular and optimized single marker system for generating Trypanosoma brucei cell lines expressing T7 RNA polymerase and the tetracycline repressor. Open Biol. 2012;2. doi:10.1098/rsob.110037

27. Wirtz $\mathrm{E}$, Clayton $\mathrm{C}$. Inducible gene expression in trypanosomes mediated by a prokaryotic repressor. Science. 1995;268: 1179-1183. doi:10.1126/science.7761835 28. Schumann Burkard G, Jutzi P, Roditi I. Genome-wide RNAi screens in bloodstream form trypanosomes identify drug transporters. Mol Biochem Parasitol. 2011;175: 91-94. doi:10.1016/j.molbiopara.2010.09.002

29. Heldin C-H, Johnsson A, Wennergren S, Wernstedt C, Betsholtz C, Westermark B. A human osteosarcoma cell line secretes a growth factor structurally related to a homodimer of PDGF A-chains. Nature. 1986;319: 511-514. doi:10.1038/319511a0

30. Wickstead B, Ersfeld K, Gull K. Targeting of a tetracycline-inducible expression system to the transcriptionally silent minichromosomes of Trypanosoma brucei. Mol Biochem Parasitol. 2002;125: 211-6.

31. Schnarwiler F, Niemann M, Doiron N, Harsman A, Käser S, Mani J, et al. Trypanosomal 
TAC40 constitutes a novel subclass of mitochondrial $\beta$-barrel proteins specialized in mitochondrial genome inheritance. Proc Natl Acad Sci. 2014;111: 7624-7629.

doi:10.1073/pnas.1404854111

32. Florimond C, Sahin A, Vidilaseris K, Dong G, Landrein N, Dacheux D, et al. BILBO1 Is a Scaffold Protein of the Flagellar Pocket Collar in the Pathogen Trypanosoma brucei. PLoS Pathog. 2015;11. doi:10.1371/journal.ppat.1004654

33. Panavas T, Sanders C, Butt TR. SUMO fusion technology for enhanced protein production in prokaryotic and eukaryotic expression systems. Methods Mol Biol Clifton NJ. 2009;497: 303-317. doi:10.1007/978-1-59745-566-4_20

34. Peroutka lii RJ, Orcutt SJ, Strickler JE, Butt TR. SUMO fusion technology for enhanced protein expression and purification in prokaryotes and eukaryotes. Methods Mol Biol Clifton NJ. 2011;705: 15-30. doi:10.1007/978-1-61737-967-3_2

35. Bastin P, Bagherzadeh Z, Matthews KR, Gull K. A novel epitope tag system to study protein targeting and organelle biogenesis in Trypanosoma brucei. Mol Biochem Parasitol. 1996;77: 235-239. doi:10.1016/0166-6851(96)02598-4

36. Gambarotto D, Hamel V, Guichard P. Ultrastructure expansion microscopy (U-ExM). Methods in Cell Biology. Elsevier; 2020. p. S0091679X20301242.

doi:10.1016/bs.mcb.2020.05.006

37. Dacheux D, Landrein N, Thonnus M, Gilbert G, Sahin A, Wodrich H, et al. A MAP6Related Protein Is Present in Protozoa and Is Involved in Flagellum Motility. PLoS One. 2012;7: e31344. doi:10.1371/journal.pone.0031344

38. Kabsch W. XDS. Acta Crystallogr D Biol Crystallogr. 2010;66: 125-132. doi:10.1107/S0907444909047337

39. McCoy AJ, Grosse-Kunstleve RW, Adams PD, Winn MD, Storoni LC, Read RJ. Phaser crystallographic software. J Appl Crystallogr. 2007;40: 658-674.

doi:10.1107/S0021889807021206

40. Emsley P, Cowtan K. Coot: model-building tools for molecular graphics. Acta Crystallogr D Biol Crystallogr. 2004;60: 2126-2132. doi:10.1107/S0907444904019158

41. Afonine PV, Grosse-Kunstleve RW, Echols N, Headd JJ, Moriarty NW, Mustyakimov M, et al. Towards automated crystallographic structure refinement with phenix.refine. Acta Crystallogr D Biol Crystallogr. 2012;68: 352-367. doi:10.1107/S0907444912001308 42. Woods A, Sherwin T, Sasse R, MacRae TH, Baines AJ, Gull K. Definition of individual components within the cytoskeleton of Trypanosoma brucei by a library of monoclonal antibodies. J Cell Sci. 1989;93 ( Pt 3): 491-500.

43. McNicholas S, Potterton E, Wilson KS, Noble MEM. Presenting your structures: the CCP4mg molecular-graphics software. Acta Crystallogr D Biol Crystallogr. 2011;67: 386-394. doi:10.1107/S0907444911007281 
Table 1. Data collection and refinement statistics

\begin{tabular}{ll}
\hline Data collection & \\
Space group & $\mathrm{C} 222_{1}$ \\
Wavelength $(\AA)$ & 0.976 \\
Cell dimensions & \\
$\quad a, b, c(\AA)$ & $60.42,73.39,120.51$ \\
Resolution $(\AA)$ & $50.0-1.87(1.94-1.87)$ * \\
Total reflections & $271,309(15,723)$ \\
Unique reflections & $21,911(1,295)$ \\
Multiplicity & $12.4(9.7)$ \\
$\mathrm{R}_{\text {-merge }}$ & $0.125(2.359)$ \\
$\mathrm{R}_{\text {-meas }}$ & $0.130(2.477)$ \\
$\mathrm{R}_{\text {-pim }}$ & $0.037(0.727)$ \\
CC(1/2) & $0.999(0.742)$ \\
CC & $1.000(0.923)$ \\
Mean I / $(I)$ & $12.76(0.77)$ \\
Completeness $(\%)$ & $95.4(57.7)$ \\
Wilson B-factor & 39.35
\end{tabular}

\section{Refinement}

Resolution $(\AA)$

20-1.87

Number of reflections

21,481

$R_{\text {work }} / R_{\text {free }}(\%)$

No. atoms

Protein

Water

82

Ligand/ion

36

$B$-factors

Protein

52.0

Water

45.2

Ligand/ion

77.8

R.m.s. deviations

$\begin{array}{ll}\text { Bond lengths }(\AA) & 0.007 \\ \text { Bond angles }\left({ }^{\circ}\right) & 0.810\end{array}$

Ramachandran plot

$\begin{array}{ll}\text { Favored (\%) } & 99.07 \\ \text { Allowed (\%) } & 0.93 \\ \text { Outlier (\%) } & 0\end{array}$

*Values in parentheses are for the highest resolution shell. 


\section{Figure legends}

Fig 1. A. Schematic representation of the BILBO1, FPC4, and BILBO2 secondary structures and $\mathrm{Y2H}$ interaction tests. A. The BILBO1 domains T3 (aa 171-587) and T4 (aa 251-587) were previously described in [10]. BILBO2 is presented as three domains: T1 (aa 1-103), T2 (aa 1150), and the BILBO1-binding domain, B1BD (aa 151-271). B. Y2H assay with full-length or domains of BILBO2 as bait and BILBO1 as prey tested on minus histidine medium (-HIS). Loading control was on medium plus histidine. Positive control involved p53 and T-antigen, whereas negative control involved Lamin and T-antigen.

Fig 2. Analysis of BILBO1 and BILBO2 interaction in a heterologous system. A. U-2 OS whole cells expressing BILBO1 (a), HABILBO2 (b), or co-expressing BILBO1 with ${ }_{\mathrm{HA}} \mathrm{BILBO2}$ (c) or with HABILBO2-T1 (d), T2 (e), BILBO2-B1BD (f) domains, and processed for immunofluorescence. B. Immunolabeling on detergent-extracted U-2 OS cells co-expressing ${ }_{\mathrm{HA}} \mathrm{BILBO} 2$ and $\mathrm{BILBO} 1_{\mathrm{GFP}}$ (a), BILBO1-T3 GFP $_{\text {(b), BILBO1-T4 }}$ GFP (c), and BILBO1-T3 GFP with HABILBO2-B1BD (d). C. Immunolabeling on detergent-extracted U-2 OS cells expressing BILBO1 mutated on the EFhands (mEFh1, mEFh2, mEFH1+2, a-c), deleted of the Efh1+2 domain (d), and co-expressed with ${ }_{\mathrm{HA}} \mathrm{BILBO2}(\mathrm{e}-\mathrm{h})$. Scale bars, $10 \mu \mathrm{m}$.

Fig 3: Cellular localization of BILBO2. A. Immunolabelling on detergent-extracted PCF cells using anti-BILBO1 and anti-BILBO2 antibodies. B. Immunolabelling on detergent-extracted PCF

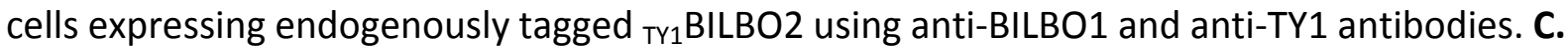
Quantification of BILBO1 and ${ }_{\mathrm{TY} 1} \mathrm{BILBO} 2$ labelling during PCF cell cycle. Error bars in C represent the standard error from three independent experiments. D. Ectopic expression of BILBO2 ${ }_{\mathrm{HA}}$ and domains was induced for $24 \mathrm{H}$ with $1 \mu \mathrm{g} / \mathrm{mL}$ of tetracycline followed by immunofluorescence on whole cells and detergent-extracted cells (Cytoskeleton). Faint but consistent labelling of BILBO2-T2 $2_{\mathrm{HA}}$ the FPC on cytoskeleton is indicated by the asterisk. Scale bars in $A, B$ and $D$ represent $5 \mu \mathrm{m}$, and $1 \mu \mathrm{m}$ (insets).

Fig 4: Depletion of BILBO1 induces cytosolic localization of BILBO2. A. Comparative growth curves between WT cells and cells expressing ${ }_{\mathrm{TY} 1 \mathrm{BILBO} 2}$ and non-induced (NI) or induced (I) for BILBO1 RNAi. B. Immunolabelling of BILBO1 and ${ }_{\mathrm{TY} 1 \mathrm{BILBO} 2}$ on BILBO1 RNAi non-induced 
(NI) or induced $24 \mathrm{H}$ and $48 \mathrm{H}$ whole cells. C. Western-blot analysis of the fate of BILBO2 during BILBO1 RNAi in whole-cell (WC) and detergent-extracted samples (CSK). Anti-enolase and antitubulin were used as detergent extraction and loading controls, respectively. D. Quantification of the Western-blot in C. Error bars represent the standard error from three independent experiments. Scale bars in B represent $5 \mu \mathrm{m}$.

Fig 5. BILBO1 and BILBO2 share a conserved N-terminal domain with conserved residues. A. Alignment of the BILBO1 and BILBO2 NTD domains. Asterisks indicate identical residues; columns indicate conserved substitution; periods indicate semi-conserved substitutions. B. Immunolocalization of chimeric BILBO1-BILBO2 proteins. Anti-BILBO1 labels both BILBO1 and

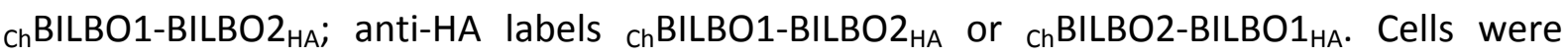
induced $18 \mathrm{~h}$ with $1 \mu \mathrm{g} \cdot \mathrm{mL}^{-1}$ tetracycline. Scale bars represent $5 \mu \mathrm{m}$.

Fig 6. Interaction between BILBO2 and FPC4 is similar to that of BILBO1 and FPC4. A. Y2H interaction assay between BILBO2 and FPC4 and domains. B. Immuno-colocalisation of ${ }_{T Y 1}$ FPC4 and BILBO2 in detergent-extracted PCF cells. C. Immunolocalization of BILBO1, ${ }_{m y c} B I L B O 2$ and ${ }_{T Y 1} F P C 4$ using U-ExM in detergent-extracted PCF cells. D. Expression in U-2 OS cell and immunolocalization of FPC4 $4_{\text {GFP }}$ and FPC4 deleted of its B1BD (FPC4- $\triangle B 1 B D_{\text {GFP }}$ ) and ${ }_{\mathrm{HA}} \mathrm{BILBO} 2$ and domains demonstrating that the BILBO2-T1 (aa 1-103) domain is not sufficient for a stable interaction between BILBO2 and FPC4 whereas a longer domain (BILBO2-T2) is stabilizing the interaction. Cells were detergent-extracted before the IF to reduce the FPC4 and BILBO2 cytosolic labelling. E. A tripartite interaction is demonstrated in U-2 OS cells by the co-labelling of FPC4 and BILBO2 and domains onto the BILBO1 polymers. Scale bars in B and $C$ represent $5 \mu \mathrm{m}$ and $1 \mu \mathrm{m}$ in insets, respectively. Scale bars in D and $E$ represent $10 \mu \mathrm{m}$. F. Schematic representation of the interactions between BILBO1, BILBO2 and FPC4.

Fig 7. Crystal structure the BILBO2-NTD/FPC4-B1BD complex. A. Superposition of the two copies of BILBO2-NTD in the asymmetric unit cell of the crystal lattice (blue and light blue), together with BILBO1-NTD (pink, 6SJQ.pdb). Shown in yellow and orange are polypeptides of FPC4 bound to BILBO2-NTD. B. Ribbon diagram of the structure of the BILBO2-NTD/FPC4 
complex in two orthogonal views. The structure of BILBO2-NTD is colour-ramped from blue to red at the $\mathrm{N}$ - and C-termini, respectively. FPC4 is shown as a yellow tube. C. Crystal structure of the complex with FPC4 residues depicted as sticks and BILBO2-NTD as an electrostatic surface plot. D. Stereo view of the $2 F_{o}-F_{c}$ map (purple) around the binding interface between FPC4 and BILBO2 contoured at $1.5 \sigma$ level. BILBO2 and FPC4 are coloured in green and yellow, respectively. All visible residues of FPC4 (aa 432-438) and two interface aromatic residues of BILBO2 (F63, W70) are labelled. Plots in (A) and (B) were generated using PyMOL (The PyMOL Molecular Graphics System, Version 1.2r3pre, Schrödinger, LLC.), the one in (C) was done by CCP4mg Presenting your structures: the CCP4mg molecular-graphics software [43], and that in (D) by COOT [40].

Fig 8. Identification of key residues involved in the BILBO2-FPC4 interaction. A. Interaction between FPC4-B1BD and BILBO2-NTD. FPC4 residues are depicted as sticks in yellow, whereas BILBO2-NTD is shown as an electrostatic surface plot. B. ITC experiments using purified BILBO2-NTD and wild-type (WT) and mutants of FPC4-B1BD. C. Co-immunolocalization of HABILBO2 (magenta) and of mutated forms of FPC4 ${ }_{\text {GFP }}$ (yellow) in U-2 OS cells (a). Coimmunolocalization of $\mathrm{FPC}_{\text {GFP }}$ (yellow) and mutated forms of ${ }_{\mathrm{HA}} \mathrm{BILBO} 2$ (magenta) (b). Scale bars, $10 \mu \mathrm{m}$.

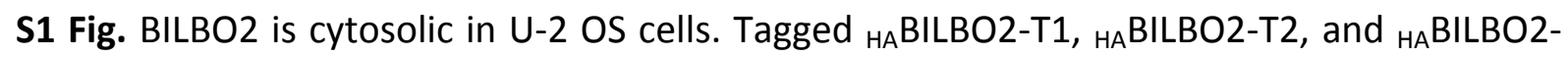
B1BD were expressed in $\mathrm{U}-2$ OS cells and immunolabelled using anti-HA and anti-BILBO1 on fixed whole cells. Scale bars, $10 \mu \mathrm{m}$.

S2 Fig. Cellular localization of BILBO2 during the T. brucei BSF cell cycle. A. Growth curves of PCF and BSF expressing endogenous tagged ${ }_{\text {TY1 }}$ BILBO2. B. Co-immunolabelling of BILBO1 and ${ }_{\text {TY1 }}$ BILBO2 on detergent-extracted cells using anti-BILBO1 and anti-TY1 antibodies. Scale bars, $5 \mu \mathrm{m}$.

S3 Fig. Growth curves of WT PCF cells, and non-induced and induced cells for ectopic

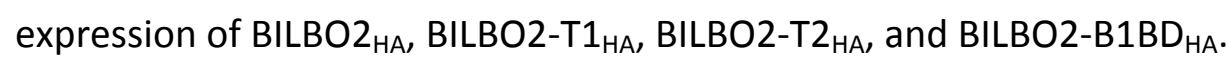


S4 Fig. BILBO2 RNAi knock-down affects neither PCF nor BSF cell growth. Growth curves and western blotting analysis of $B I L B O 2$ RNAi in ${ }_{\mathrm{T} 1} \mathrm{BILBO} 2$ expressing PCF and BSF cells. The antienolase was used as a loading control.

S5 Fig. Growth curves of WT PCF cells, and non-induced and induced cells for ectopic expression of $\mathrm{BILBO}_{\mathrm{HA}}(\mathrm{A})$ and $\mathrm{BILBO}_{\mathrm{HA}}(\mathrm{B})$ and chimeric BILBO2-BILBO1 $1_{\mathrm{HA}}(\mathrm{C})$ and BILBO1$\mathrm{BILBO}_{\mathrm{HA}}(\mathrm{D})$.

S6 Fig. A. Epifluorescence image of U-ExM triple labelling of tubulin, BILBO1 and TbSAXO, an axonemal protein. The arrowheads indicate the MTQ. B. 3D rendering of confocal analysis of U-ExM co-labelling of BILBO1 and ${ }_{\text {myc }} \mathrm{BILBO} 2$ that were used to generate S7 movie.

S7 movie. Movie of 3D rendering after confocal analysis of BILBO1 (magenta) and mycBILBO2 (yellow) co-labelling.

S8 Fig. Interaction between BILBO2-NTD and FPC4-B1BD. A. Zoom-in view of the central part of the interface between BILBO2 and FPC4 with the $2 F_{o}-F_{c}$ map (grey) contoured at $1.5 \sigma$ level. An ordered water molecule form multiple hydrogen bonds with residues from both proteins.

B. Details of the interaction network between BILBO2 and FPC4. The plot was generated using DIMPLOT in the LigPlot plus suite. 
A

BILBO1

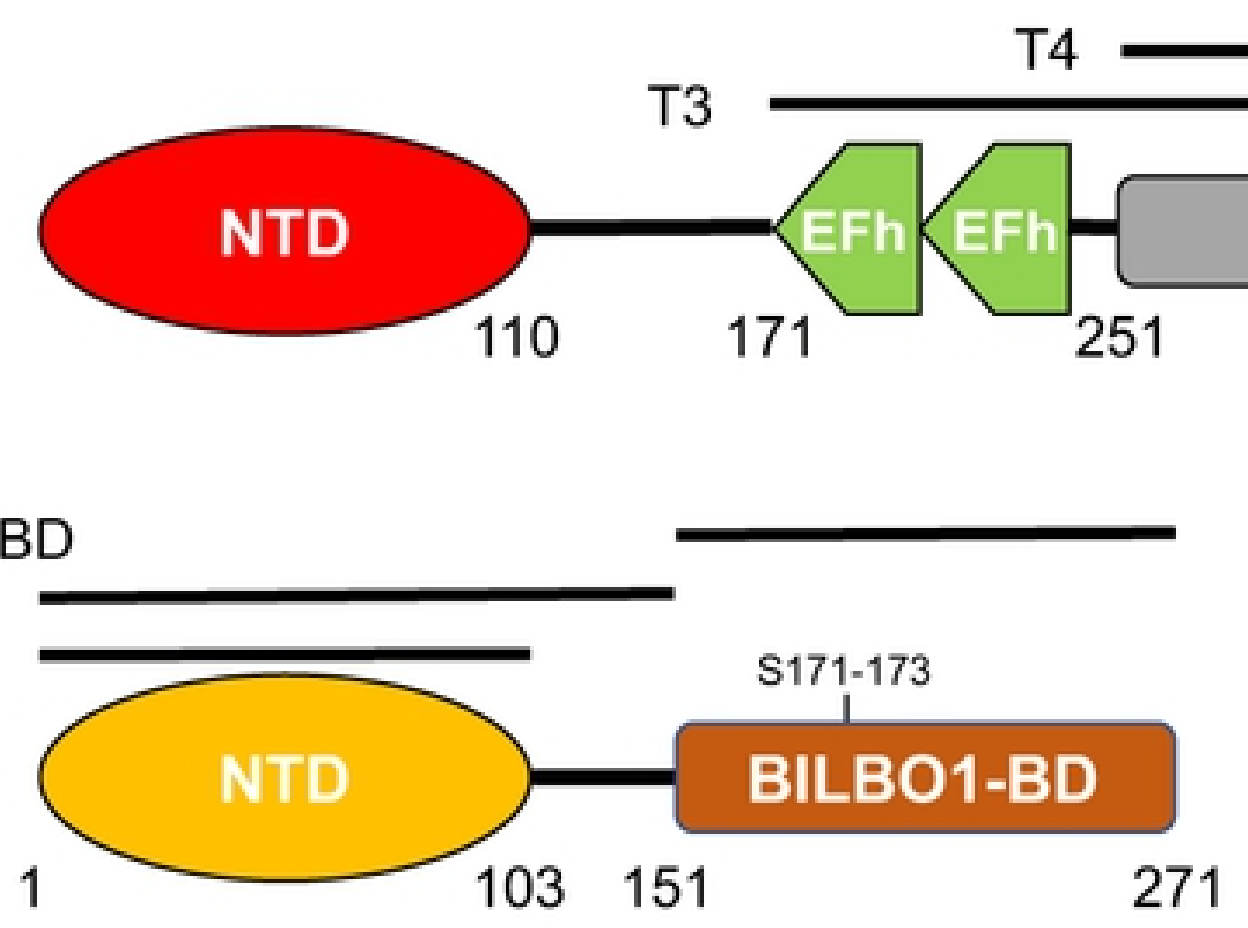

FPC4

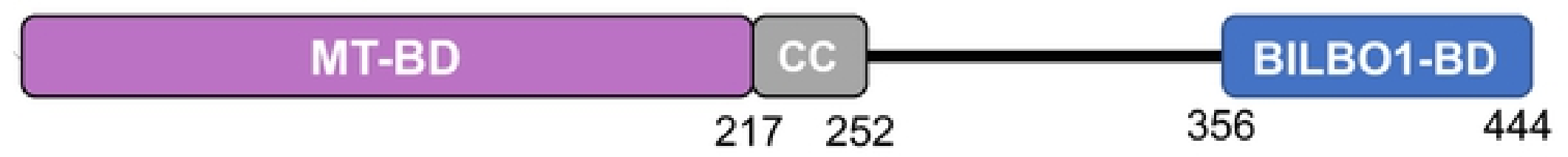

B $\quad$ BILBO2

BILBO1
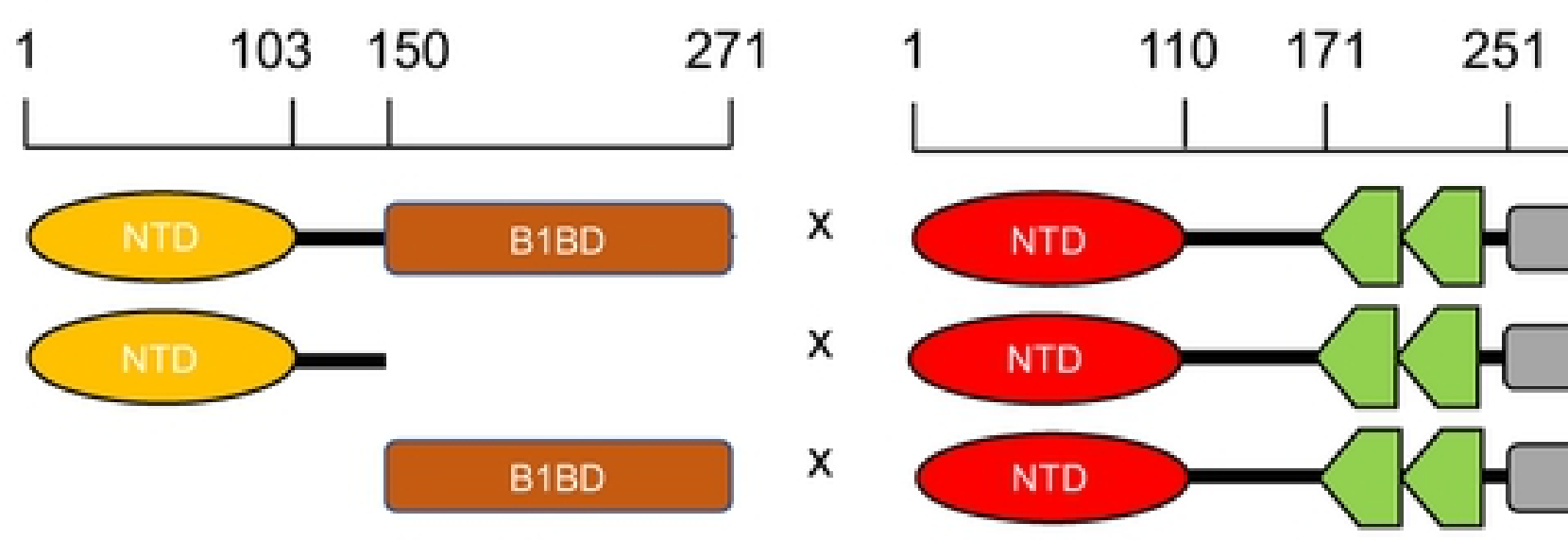

251

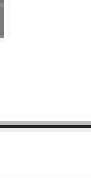

587
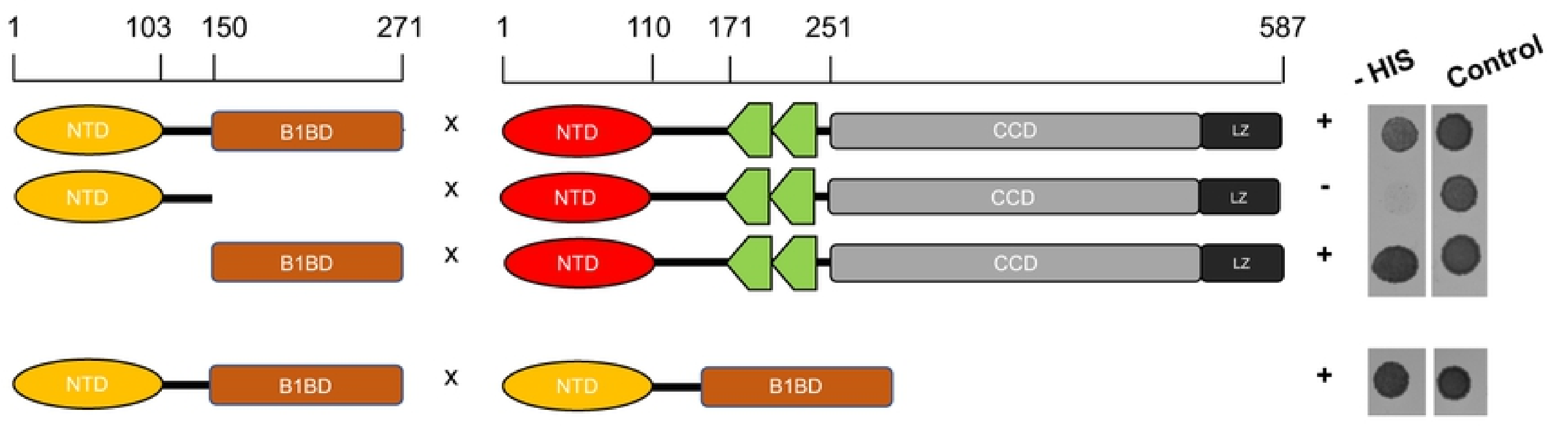

Control-

Control+

Figure 1 


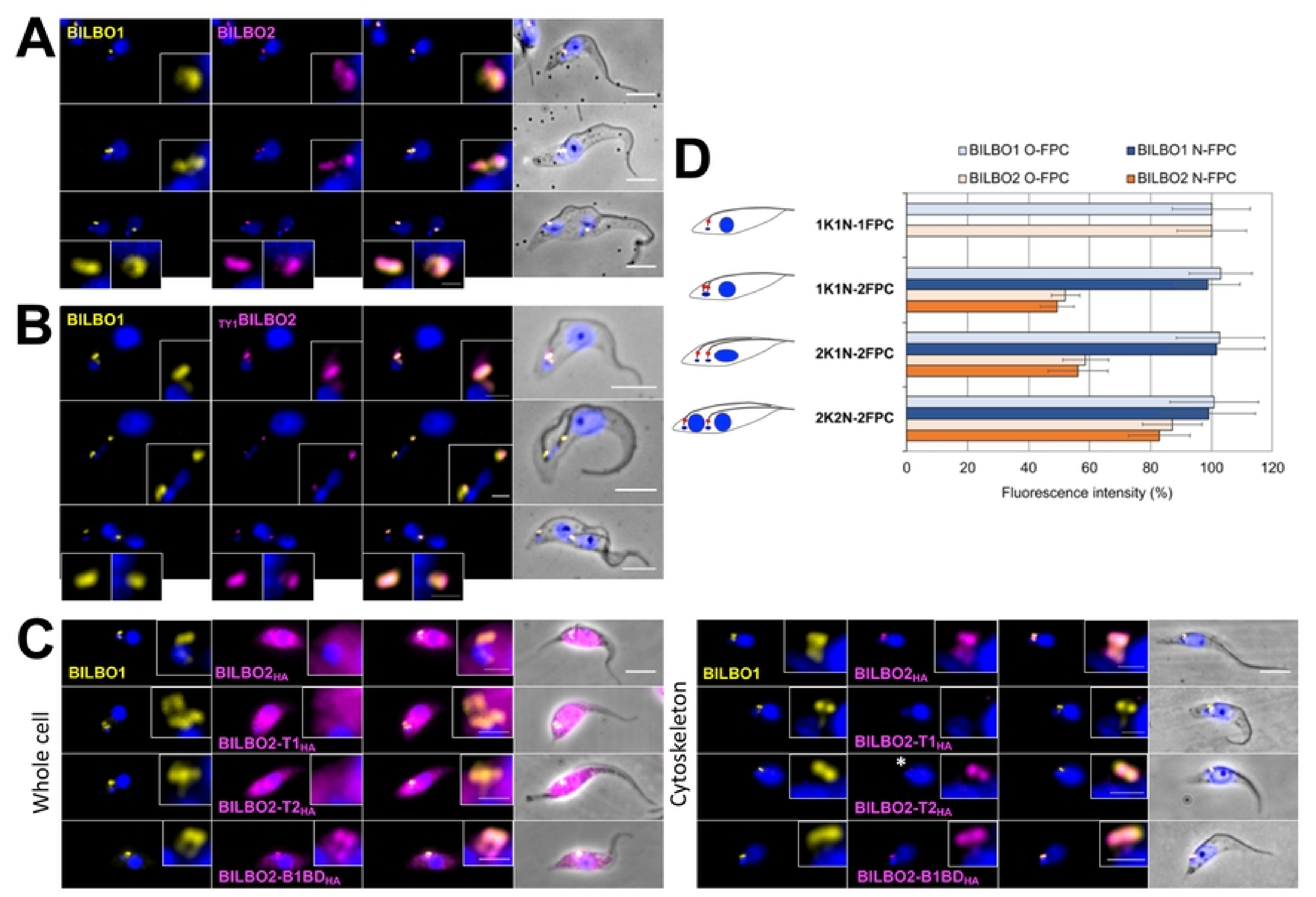

Figure 3 
A

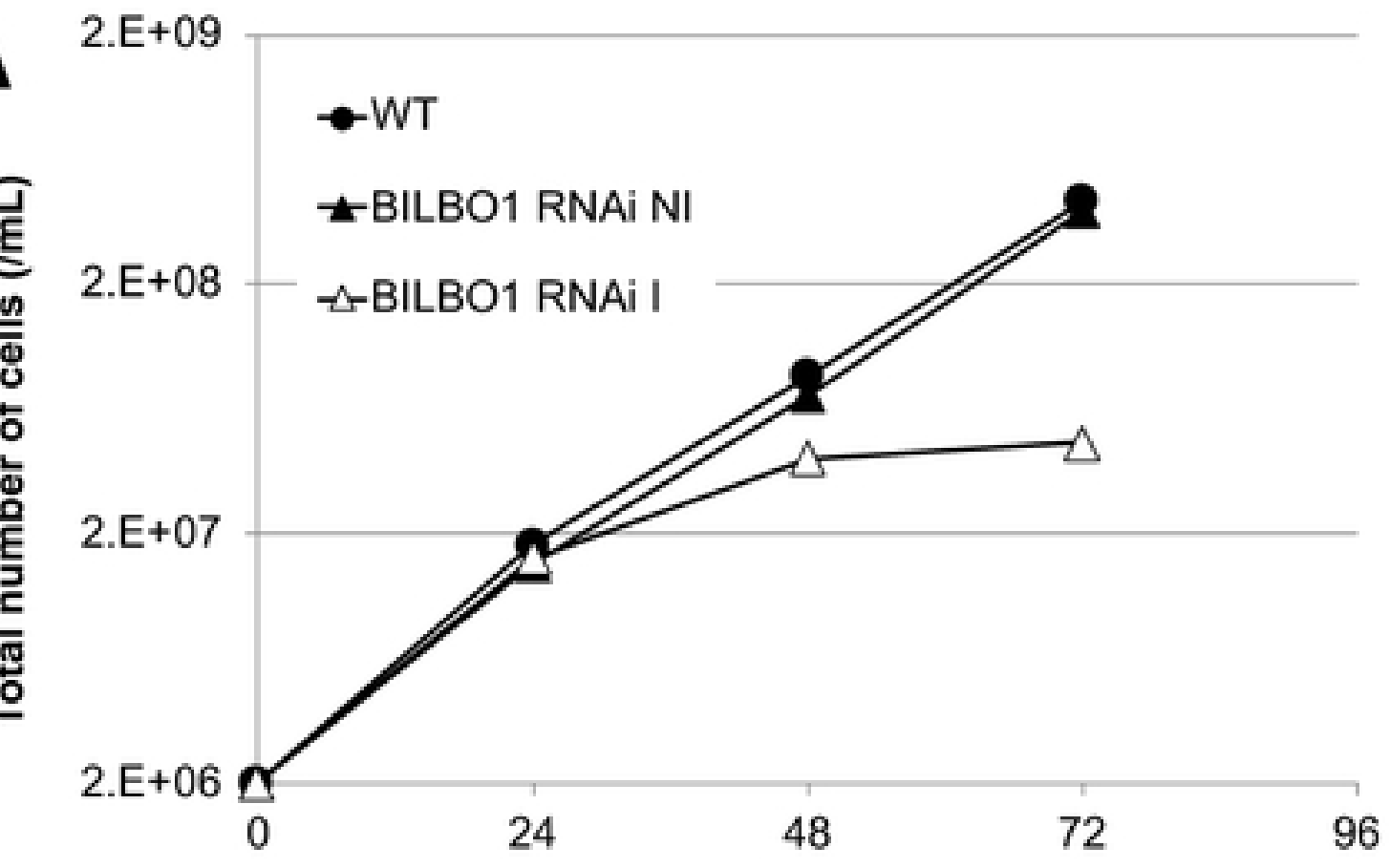

B

NI

BILBO1

Hours of induction
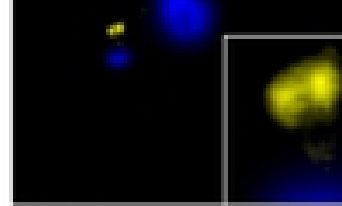

$24 \mathrm{H}$

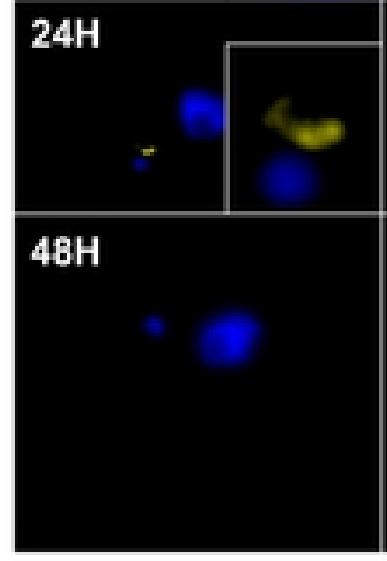

0

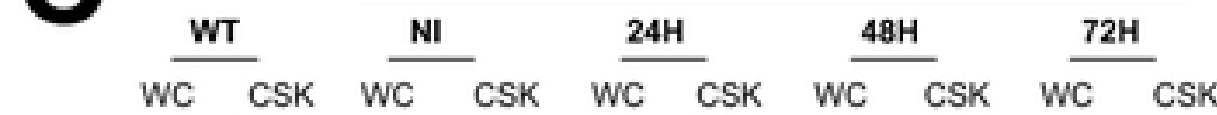
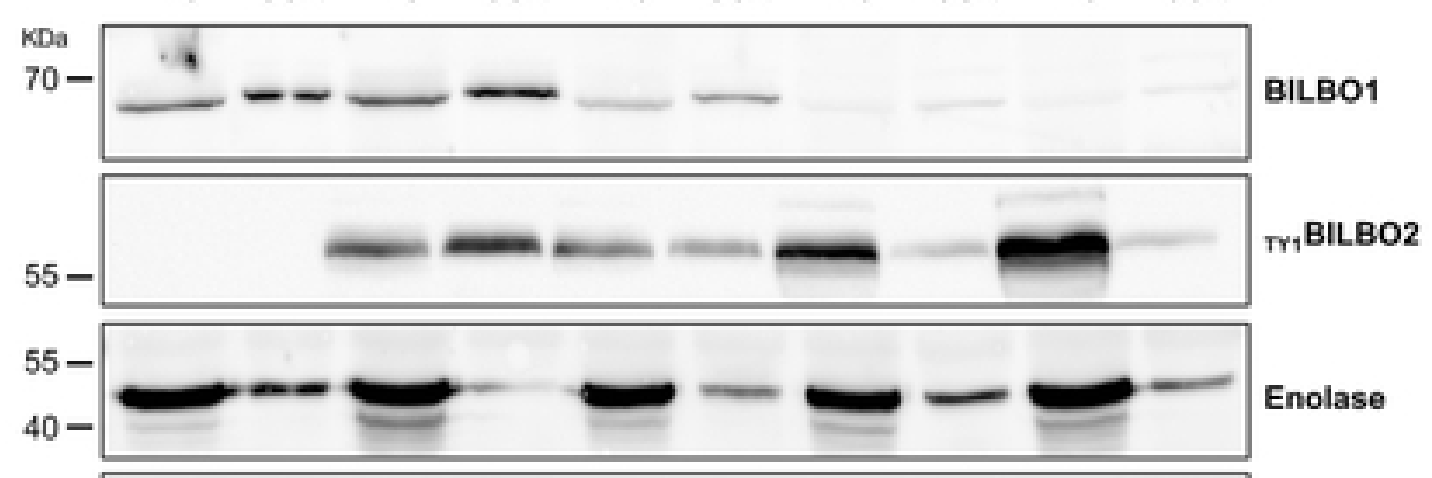

$55-$

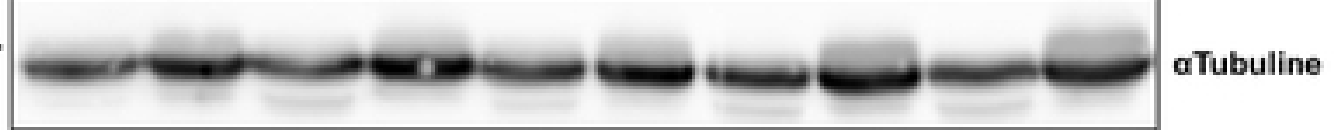

D

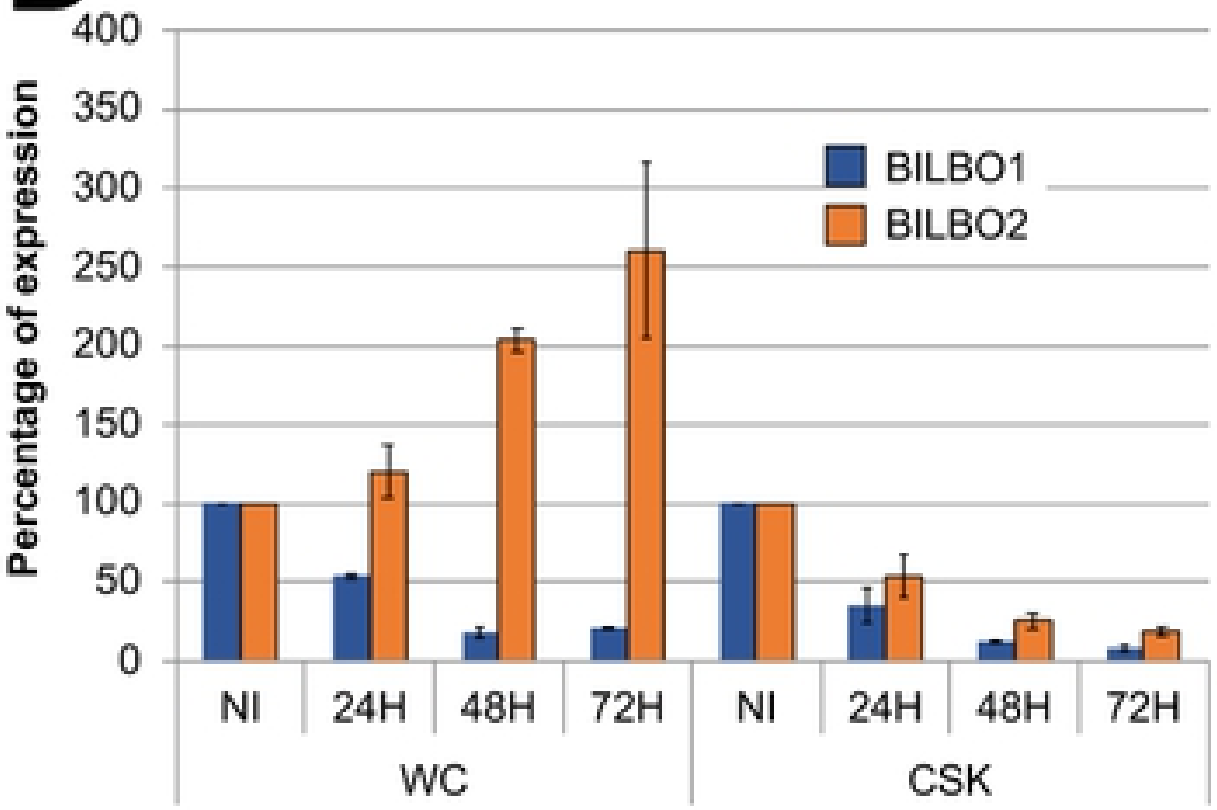

Hours of induction

Figure 4 
TbBILBO1-NTD TbBILBO2-NTD --MAFLVQVAADIFNNKVNFELSFP-SRPSISELTRSAETAFSNEISLRRPDNVPSHKFH MMGGISICVATDCDGEKVNLRFLFDAAGPSVSRLLNYSTTAFNNYFRLKGI----SRAFA

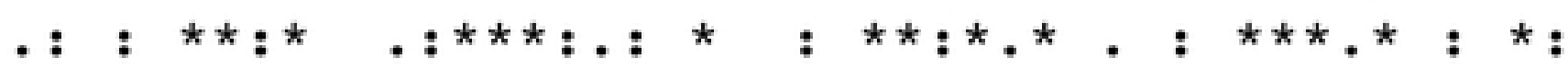
*: *

TbBILBO1-NTD TbBILBO2-NTD

SSKIKMYDEELNKWVDLIREDQLTDYCQLYVFQPPNEWHKESQKEI PPAMKP---VNSAVVFNDVHCTWDRLERTTQLLHNSQVYLFQPDTL DIPAAIPEPYEG .. : : : *

$\star * . . *: *: * *$. : ** * :

B

ChBILBO1-BILBO2

ChBILBO2-BILBO1
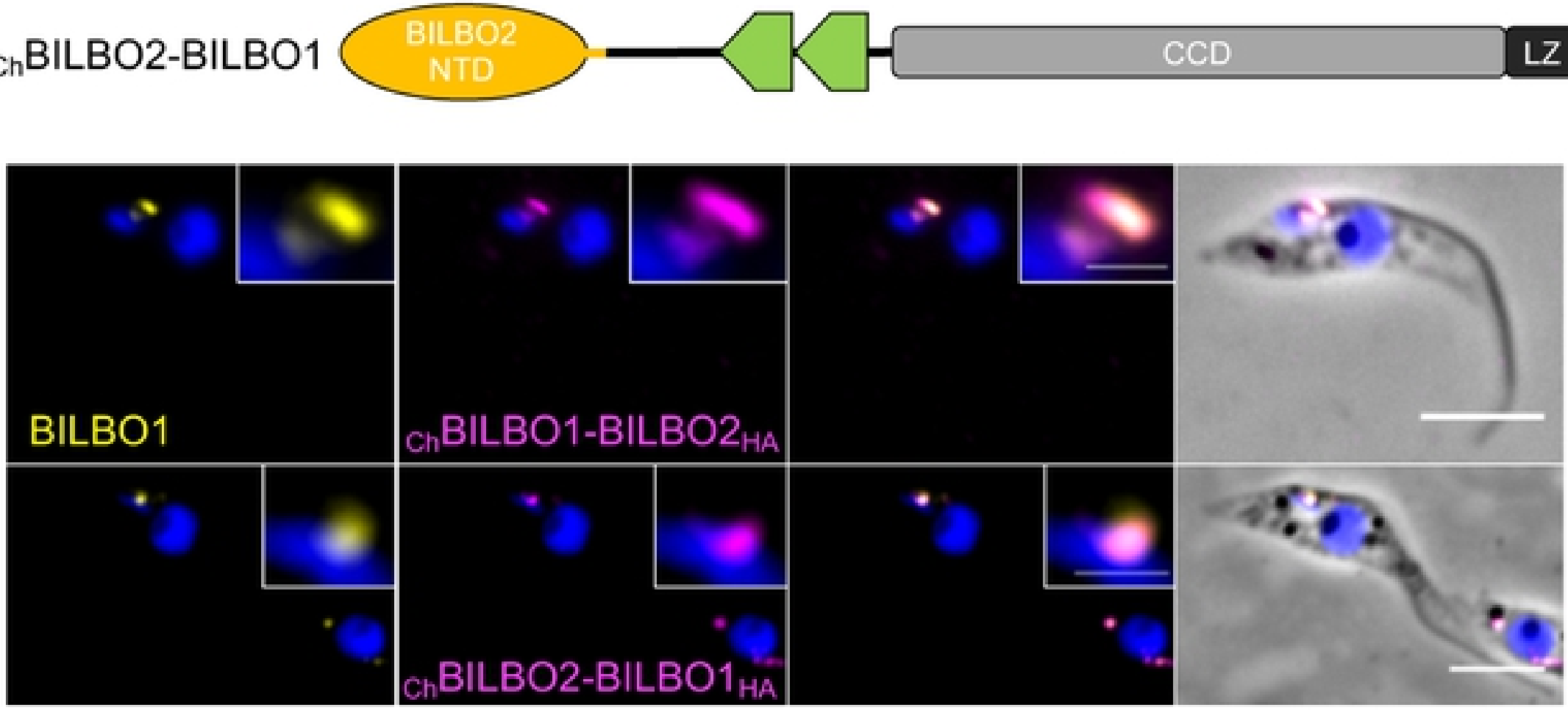

Figure 5 

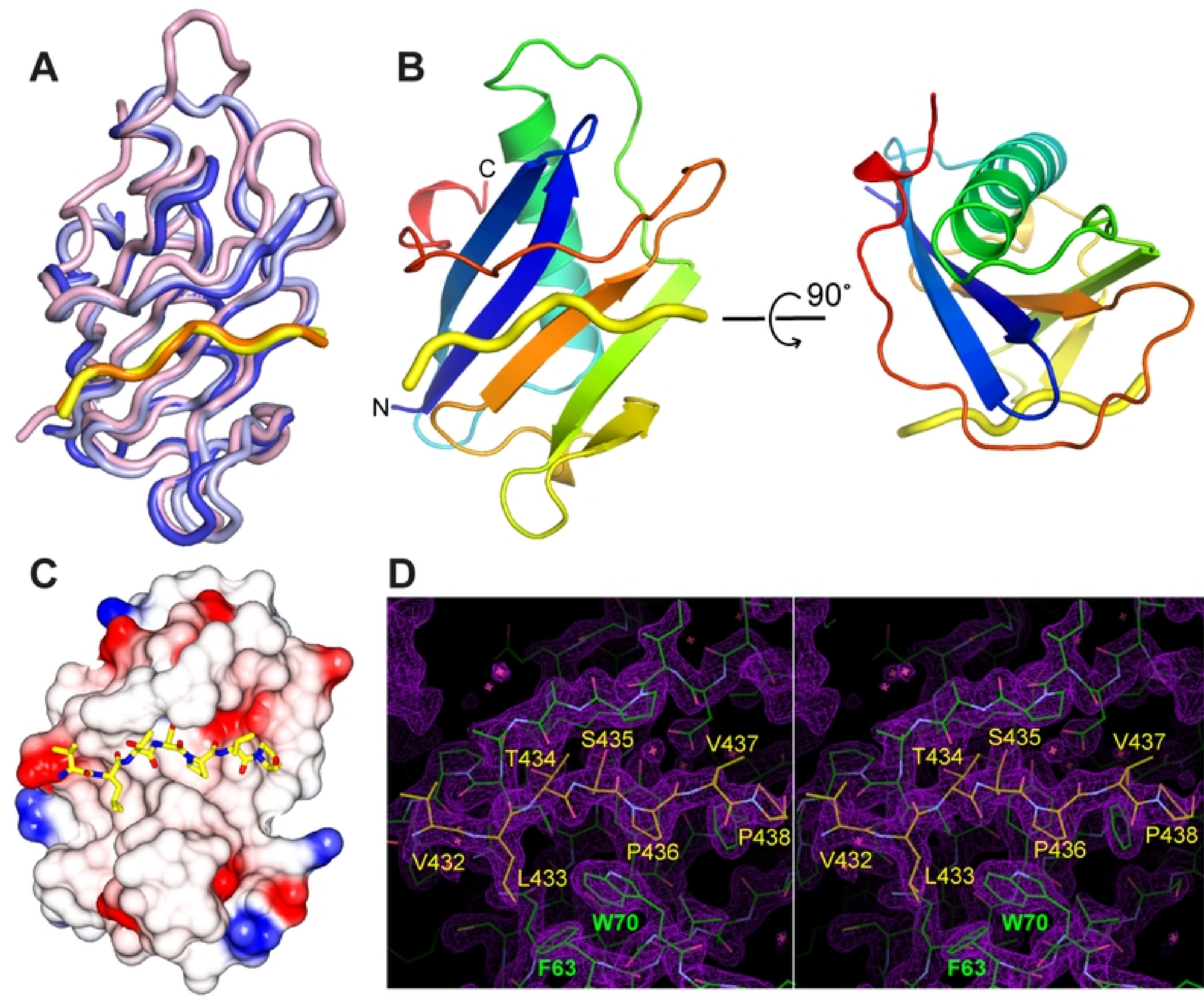

D

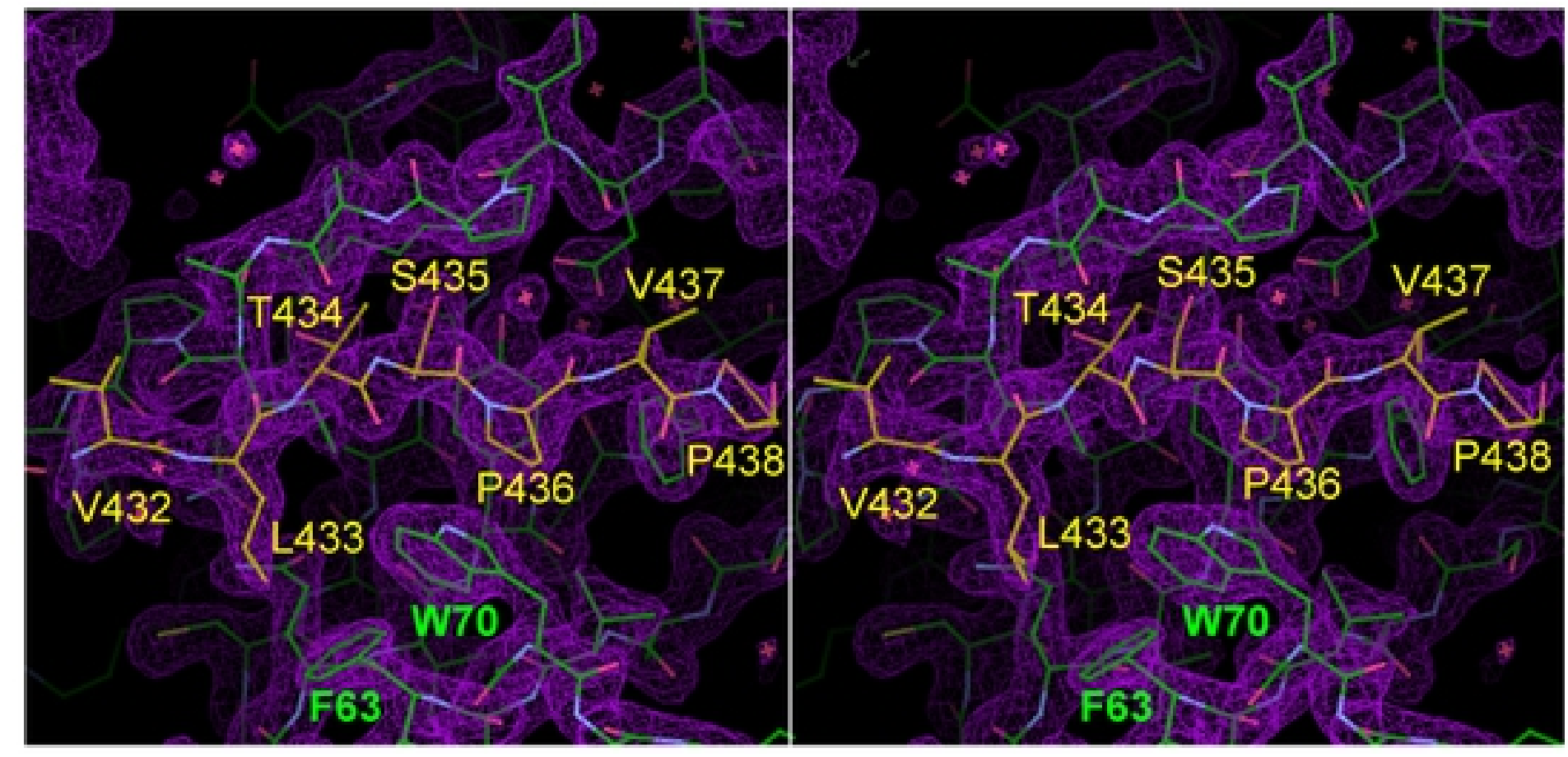

Figure 7 


\section{A}

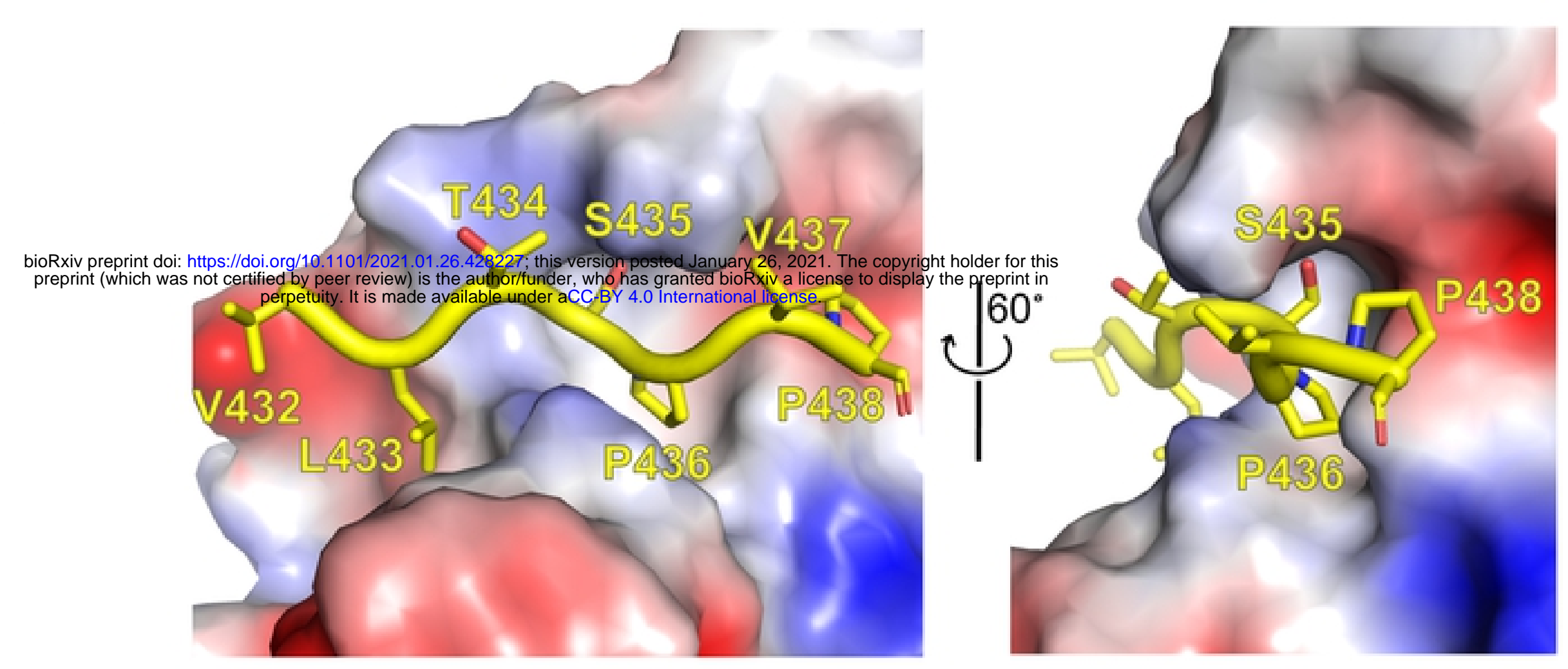

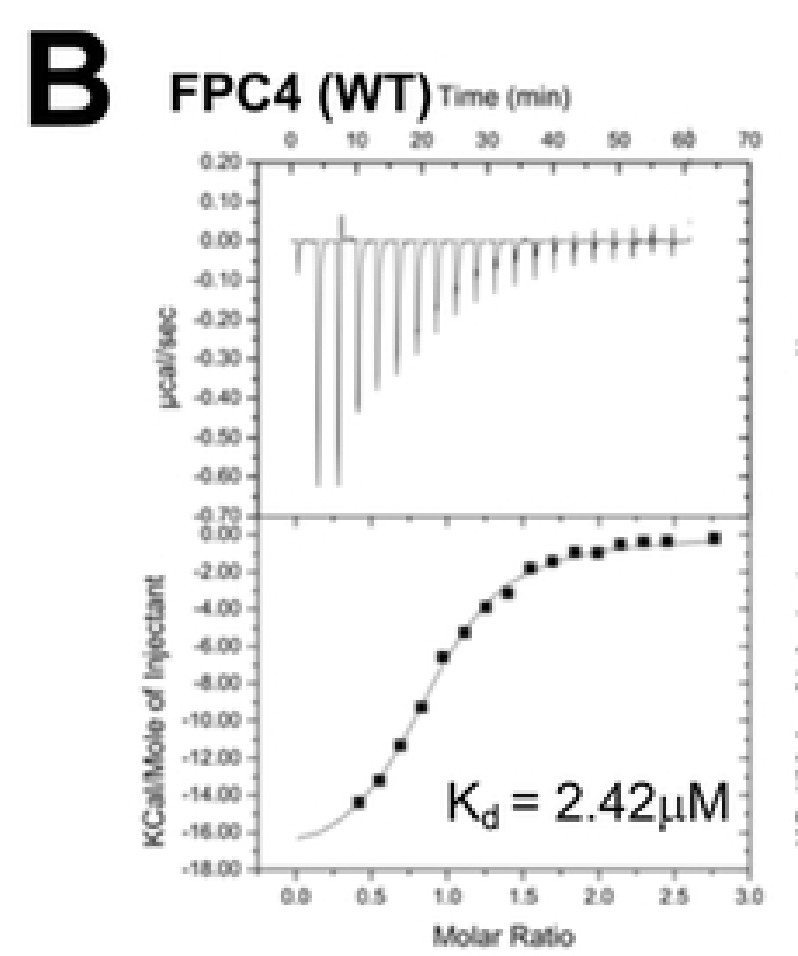

$\mathrm{S} 435 \rightarrow \mathrm{A}$ Time (min)

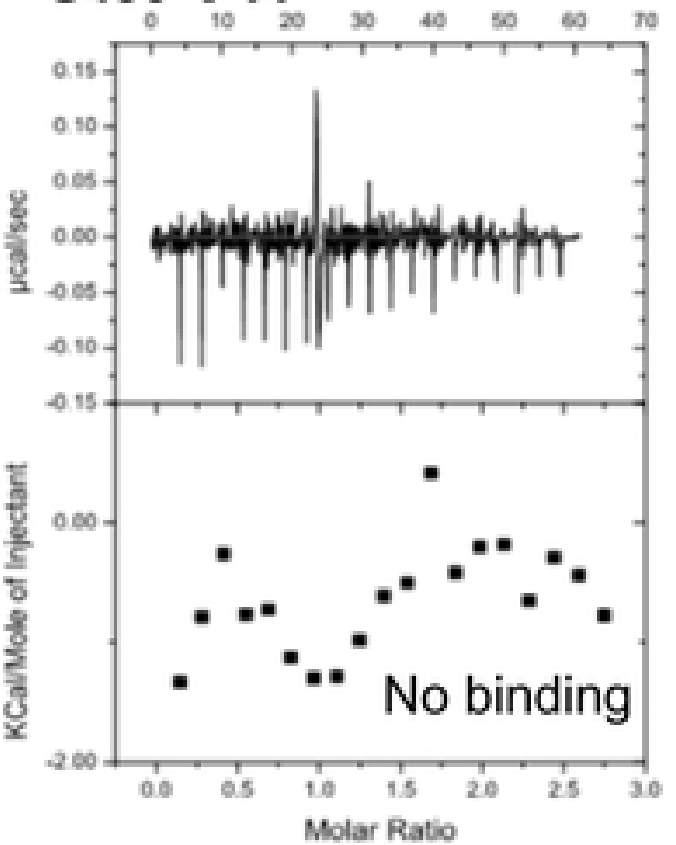

P436 $\rightarrow$ A Time(min)

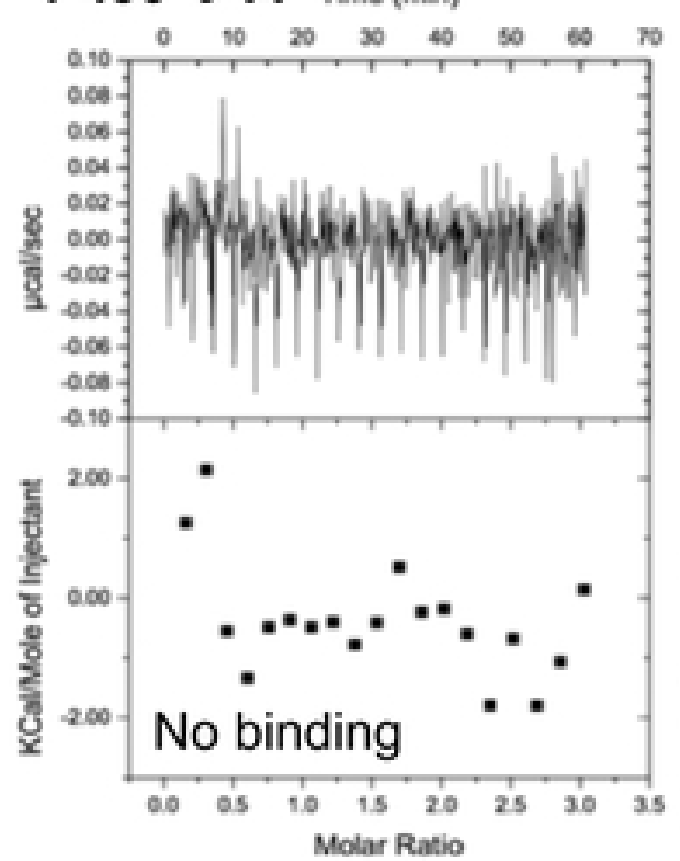

P438 $\rightarrow$ A Time(min)

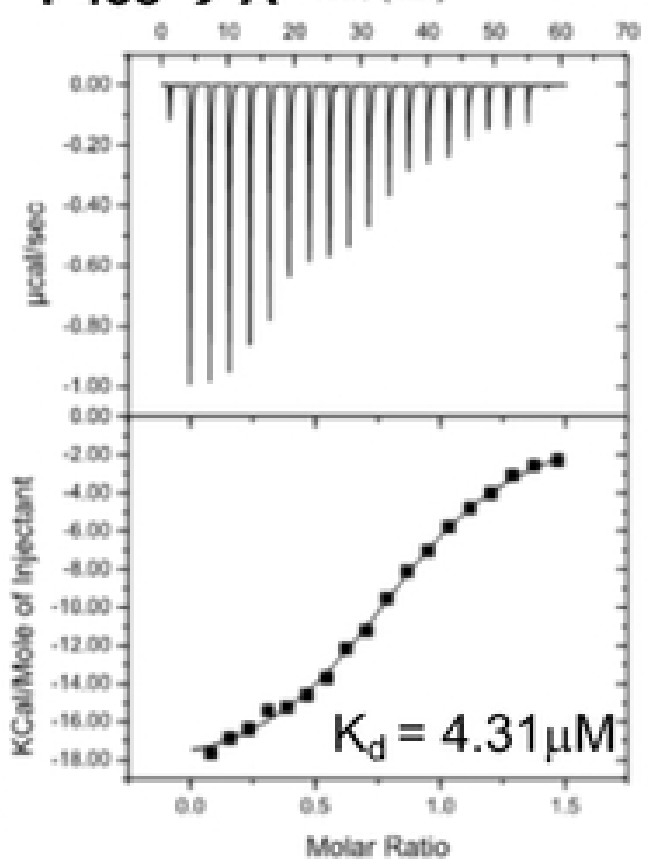

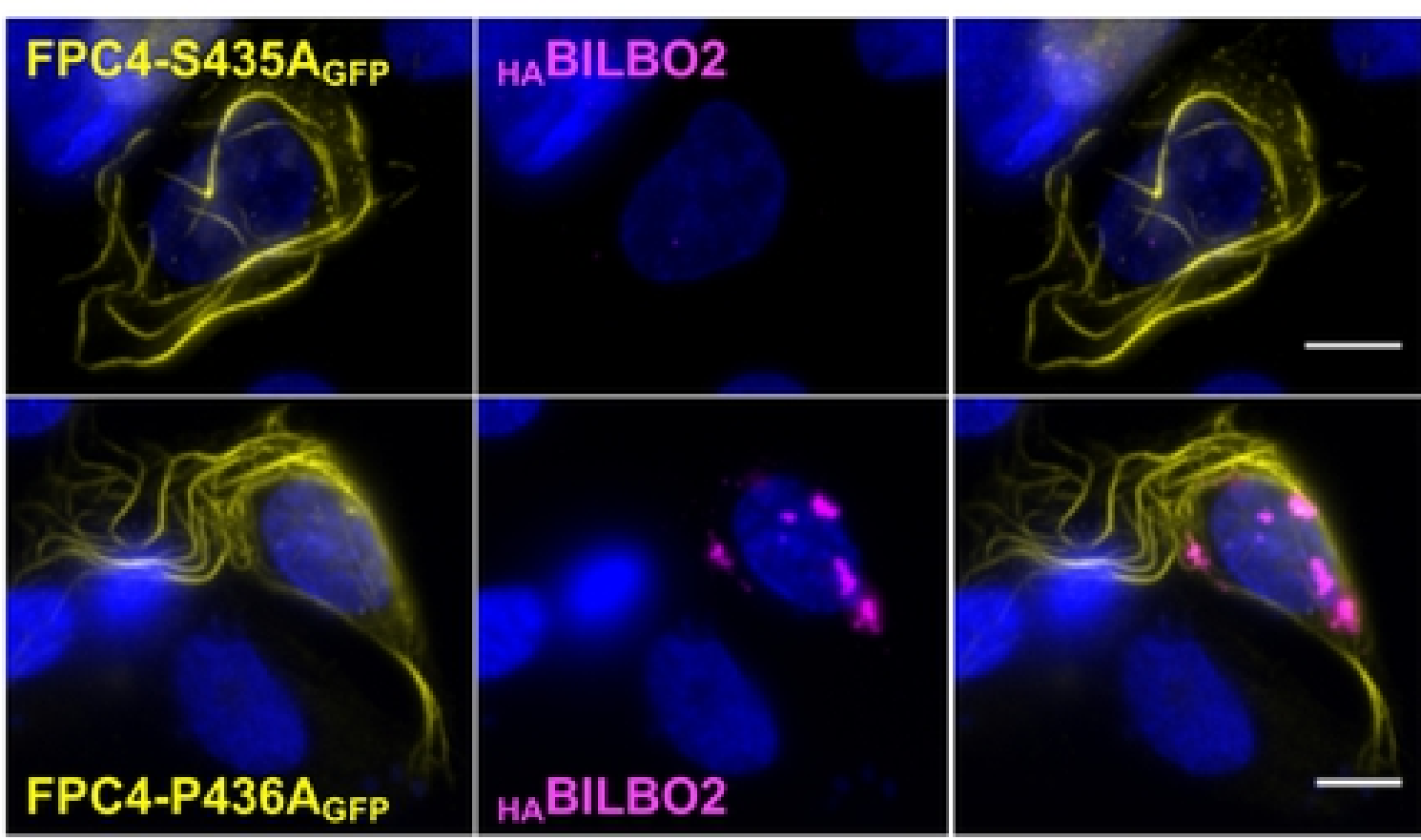

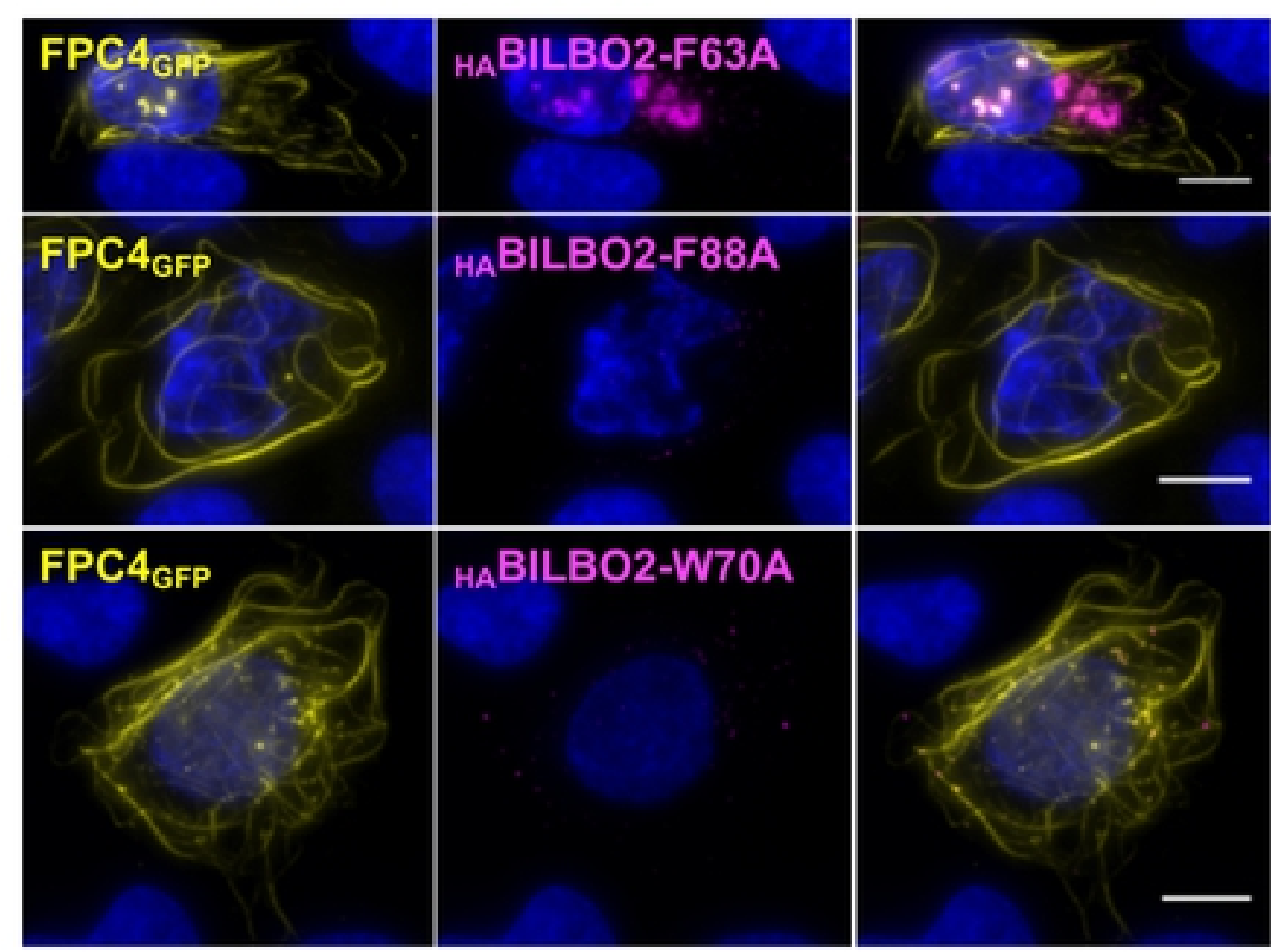

\section{Figure 8}

